\title{
Reversible and Species-Specific Depigmentation Effects of AZD3293, a BACE Inhibitor for the Treatment of Alzheimer's Disease, Are Related to BACE2 Inhibition and Confined to Epidermis and Hair
}

\author{
G. Cebers ${ }^{1}$, T. Lejeune ${ }^{2}$, B. Attalla ${ }^{2}$, M. Soderberg ${ }^{3}$, R.C. Alexander ${ }^{1}$, S. Budd Haeberlein ${ }^{1}$, A.R. Kugler ${ }^{1}$, \\ E.W. Ingersoll ${ }^{1}$, S. Platz 4 , C.W. Scott ${ }^{5}$
}

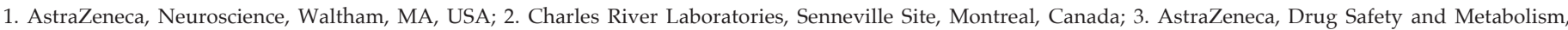
Gothenburg, Sweden; 4. AstraZeneca, Drug Safety and Metabolism, Cambridge, UK; 5. AstraZeneca, Drug Safety and Metabolism, Waltham, MA, USA

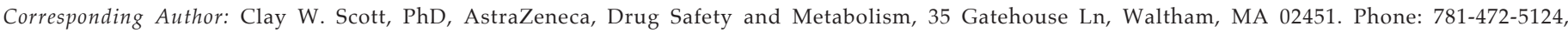
Email: clay.scott@astrazeneca.com.

J Prev Alz Dis 2016;in press

Published online 2016, in press

\begin{abstract}
BACKGROUND: AZD3293 (also known as LY3314814) is a novel, potent, non-selective BACE1/BACE2 inhibitor currently in Phase 3 clinical development for the treatment of Alzheimer's disease.

OBJECTIVES: The purpose of these studies was to characterize the effects, putative mechanism, and reversibility of hypopigmentation following treatment with AZD3293 in pigmented Long-Evans rats, Beagle dogs, human cell cultures, and humans.

DESIGN: Nonclinical studies were conducted in Long-Evans pigmented rats, and both young and older Beagle dogs using a variety of oral dose levels of AZD3293 or AZD3839 (BACE inhibition reference compound; used in older dogs only) for dosing durations of 13 to 26 weeks. In vitro studies of normal human epidermal melanocytes and reconstituted human epidermis were also conducted. Skin biopsy data from a multiple-dose Phase 1 clinical study of AZD3293 (NCT01795339) are also reported.
\end{abstract}

SETTING: Nonclinical in vivo and in vitro studies were conducted in laboratory settings in the US, Canada, and France; the multiple dose clinical study was conducted in a specialized inpatient setting in the US.

PARTICIPANTS: Beagle dogs: 13-week study N=36 young (8-10 mo) animals; 39 -week study $\mathrm{N}=64$ young animals; and a second 13-week study $\mathrm{N}=32$ older (30-32 mo) animals. Long-Evans rats: $\mathrm{N}=68$ animals. Multiple-dose clinical study: only data for subjects enrolled in Part 2 of this study are included in this report $(\mathrm{N}=16)$.

INTERVENTIONS: AZD3293 was the primary intervention used in these studies. AZD3839, a relatively BACE1-selective reference inhibitor compound was used in one group in the 13 week study in older Beagle dogs and one in vitro assessment. Finally, AZ1340, another relatively BACE1-selective reference inhibitor compound was used only in one in vitro assessment.

MEASUREMENTS: Measurements for the nonclinical studies in dogs and rats included macroscopic observation and assessment of skin biopsies via histopathology, immunochemistry, and electron microscopy. Measurements for the in vitro studies included melanocyte premelanosome protein (PMEL) processing, cytotoxicity, melanin synthesis, Pmel17 labeling, and melanocyte dendricity. Measurements in the clinical study included scoring of melanin content in skin biopsies taken before and after dosing with AZD3293 over 14 days at dose levels up to $150 \mathrm{mg}$.

RESULTS: Depigmentation in rats and dogs was limited to skin, hair, and mucosa with no effects on other pigmented tissues. At a cellular level depigmentation was observed within a week of treatment, whereas the appearance of depigmentation in skin and hair did not become apparent until, at earliest, 4 weeks of treatment. The depigmentation effects were reversible, not associated with degenerative or inflammatory changes, and were dose- and species-dependent in severity. Full recovery of melanization was observed at the microscopic (cellular) level and at least partial recovery was seen in the macroscopic appearance of animals by the end of the 12-week recovery period in both rats and dogs. Interestingly, no changes in melanin production or melanocyte morphology were seen in human primary melanocytes or reconstituted human epidermis in vitro. Finally, there were no changes in melanization level in skin biopsies following 12 days of daily AZD3293 treatment at doses of AZD3293 up to $150 \mathrm{mg} /$ day in human subjects.

CONCLUSIONS: AZD3293, a novel, potent, non-selective BACE1/BACE2 inhibitor is in development as a potentially disease-modifying treatment for Alzheimer's disease. Chronic nonclinical studies in Beagle dogs and pigmented rats showed macroscopic and microscopic hypopigmentation effects of AZD3293 that were limited to skin, hair, and mucosa. These effects were shown to be reversible in both species. Analysis of data from nonclinical and in vitro studies suggests that hypopigmentation is caused by BACE2 inhibition resulting in accumulation of a premelanosome protein fragment, which interrupts the normal production of melanin. No macroscopic or microscopic reports of hypopigmentation were observed in a Phase 1 clinical study following 13 doses of AZD3293 over 14 days at dose levels up to $150 \mathrm{mg} /$ day. These data suggest that hypopigmentation is species-specific and humans appear to be least sensitive to the depigmentation effect caused by BACE2 inhibition.

Key words: Alzheimer's disease, AZD3293, BACE1 protein-human, BACE2 protein-human, toxicology, melanins, hypopigmentation, drug evaluation, nonclinical.

Abbreviations: AZ1340: A relatively BACE1-selective inhibitor (reference compound); AZD3293: A BACE inhibitor drug in development for the treatment of Alzheimer's disease. Also known as LY3314814; AZD3839: A relatively BACE1-selective inhibitor 
(reference compound); A $\beta$ : Amyloid- $\beta$; A $\beta P P$ : Amyloid- $\beta$ protein precursor; $A D$ : Alzheimer's disease; BACE1: $\beta$-site amyloid precursor protein cleaving enzyme 1; BACE2: $\beta$-site amyloid precursor protein cleaving enzyme 2; HEE: Hematoxylin and eosin; $I C_{50}$ : 50\% inhibitory concentration; KO: Knockout; L-DOPA: Dihydroxyphenyl-alanine L3,4; LY3314814: A BACE inhibitor drug in development for the treatment of Alzheimer's disease. Also known as AZD3293; M : Fragment of PMEL that contains a disulfidelinked transmembrane domain; NHEK: Normal human epidermal keratinocytes; NHEM: NDP-MSH-stimulated melanocytes; OD: Optical density; PBO: Placebo; PMEL: Premelanosome protein (also known as PMEL17, SILV, or gp100); QD: Once daily; QW: Once weekly; RHEm: Melanocyte-containing reconstructed human epidermis; Sb: Stratum basale; Ss: Stratum spinosum.

\section{Introduction}

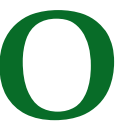
verproduction of amyloid- $\beta(A \beta)$ peptides and their accumulation in the brain are characteristic features of Alzheimer's disease $(A D)$, a neurodegenerative disease with no effective disease-modifying treatment available (1-3). Generation of $\mathrm{A} \beta$ peptides is very well described and involves sequential cleavage of $A \beta$ protein precursor $(A \beta P P)$ by Beta-site amyloid precursor protein cleaving enzyme 1 (BACE1; also known as Beta-secretase) and gammasecretase $(4,5)$. Genetic mutations in $A \beta P P$ have been linked causally to early onset $\mathrm{AD}$, and two mutations in A $\beta P P(K 670 N / M 671 L$-the Swedish mutation, and the A673T variant) have been associated with changes in BACE1 activity and confer early onset $\mathrm{AD}$ and reduced risk for AD respectively (6). BACE1 is the first step in the processing of $A \beta P P$ to $A \beta$ peptides, and its inhibition is an attractive target for therapeutic intervention to stop the production of $A \beta$ (2). In contrast, achieving $A \beta$ reduction by inhibitors of gamma-secretase activity in the CNS was associated with numerous adverse events (7). Thus, reduction of $A \beta$ production by the means of inhibiting BACE1 is currently considered to be one of the most promising directions in modifying and potentially halting the progression of $\mathrm{AD}(6,8-13)$. Several BACE1 inhibitors are currently in various stages of clinical development and initial data regarding the clinical efficacy as well as safety of this approach will be reported as soon as 2017 (14-16).

The BACE protease subfamily has two members, BACE1 and BACE2. BACE2 is structurally similar to BACE1 particularly in the catalytic center of the enzyme but has divergent tissue and intracellular distribution as well as different substrate specificity (17). The active sites of both BACE1 and BACE2 appear to be relatively conserved across mammalian species using an evolutionary tracing methodology (18). BACE2 expression levels in the brain are low and its inhibition is not considered necessary for therapeutic lowering of brain $A \beta$ levels (19). However, most if not all of the BACE inhibitors currently in clinical development, including two of the drugs furthest along in clinical development (MK-8931 and AZD3293), are not selective for BACE1 as they also inhibit BACE2, albeit with different potencies (14).

BACE2 knockout mice display a hypopigmented phenotype relative to wild-type mice $(20,21)$. Among the BACE2 substrates, premelanosome protein (PMEL, also known as PMEL17, SILV, or gp100) has drawn particular attention because of its role in black melanin (also called eumelanin) production and because a variety of pigmentation deficit phenotypes due to PMEL mutations have been observed in a number of species including mice (22-24) but curiously not in humans (25). PMEL is only expressed in pigment-generating cells $(26,27)$ that synthesize primarily eumelanin. Synthesis of red-brown melanins, also called pheomelanins, occurs by a different enzymatic pathway (28) and does not require PMEL (29). PMEL is cleaved early in the maturation of the melanin-generating organelle, the melanosome (30), to generate $\mathrm{M} \beta$, a disulfide-linked transmembrane domain containing fragment. $\mathrm{M} \beta$ is cleaved exclusively by BACE2 (21). The resulting amyloid $M \beta$ fragments form a fibrillar matrix within melanosomes upon which eumelanin is subsequently polymerized (31). BACE2 inhibition might therefore cause impaired eumelanin packaging in melanosomes due to the inability to produce the PMELderived fibril matrix (21).

While the significance of BACE2 and its PMELprocessing role in eumelanin synthesis was first described in genetically modified animals and in vitro systems, it has now been supported by data from both in vitro and vivo studies in which BACE2 activity was blocked pharmacologically. Shimshek, et al. (20) reported that wild type as well as BACE2+/- and BACE2-/- mice treated with the potent dual BACE1/BACE2 inhibitor NB-360 displayed dose-dependent, mouse strainspecific and, most importantly, seemingly irreversible fur depigmentation. In agreement with previous reports $(21,32,33)$, authors further concluded that BACE2 inhibition disturbs PMEL processing and causes insufficient melanosome maturation resulting in hair depigmentation. In this manuscript we present data from 3 species describing the species sensitivity, reversibility, and BACE2 dependency of hypopigmentation.

\section{Methods}

\section{Interventions}

The primary intervention in these studies was AZD3293 camsylate. AZD3839, a relatively BACE1selective reference inhibitor compound, was used in the 13-week study in older Beagle dogs and one in vitro (Western Blot) assessment. Finally, AZ1340, another relatively BACE1-selective reference inhibitor compound was used only in the Western Blot assessment. The 
$50 \%$ inhibitory concentrations $\left(\mathrm{IC}_{50} \mathrm{~s}\right)$ of AZD3293 for BACE1 and BACE2 are nearly identical at 0.6 and 0.9 $\mathrm{nM}$, respectively, a 1.5-fold margin of selectivity (16). By contrast, the $\mathrm{IC}_{50} \mathrm{~s}$ for BACE1/BACE2 for AZD3839 (34) and AZ1340 are 36/444 nM (a 12-fold margin) and $308 />5,000 \mathrm{nM}$ (at least a 16-fold margin), respectively (using the same methodology as (16)). In comparison to AZD3293, therefore, AZD3839 has 60-fold lower potency for BACE1 and 500-fold lower potency for BACE2, while AZ1340 has 500-fold lower potency for BACE1 and $>5500$-fold lower potency for BACE2.

\section{Animals}

All animal studies were conducted at Charles River Laboratories (CRL) Montreal (Canada) or CRL Edinburgh (UK). Animal studies were designed using current guidelines for repeat-dose toxicity studies issued by the EMEA (Europe), MHLW (Japan) and FDA (USA), and were primarily intended to assess the overall toxicity/ safety profile of AZD3293 and not solely to investigate the depigmentation phenomenon (only data pertinent to depigmentation are reported here). Young (8-9 mos of age at study start) or older (30-32 mos of age) Beagle dogs were socially housed, had free access to water, and were fed 300-350 g of chow daily throughout the study. Long Evans rats were 7 weeks of age at study start. Animals were group housed in stainless steel perforated floor cages, had unlimited access to water, and were fed ad libitum throughout the study.

\section{Studies in young (8-10 months old) dogs}

\section{3-week dog study}

Four groups of Beagle dogs, each consisting of 3 males and 3 females, were given AZD3293 orally once daily for 13 weeks at dose levels of 0 (control), 1, 3, and $30 \mathrm{mg} /$ $\mathrm{kg} /$ day. An additional 2 dogs/sex were given 0 , 3, or 30 $\mathrm{mg} / \mathrm{kg} /$ day to assess the potential reversibility of any findings following an 8-week drug-free period.

Due to adverse clinical findings considered to be dose limiting in some animals given $30 \mathrm{mg} / \mathrm{kg} /$ day, dosing at this dose level was discontinued. After a wash-out period of one week, dogs in this group were re-designated as Group 5 and were dosed for 13 weeks at the lower dose of $15 \mathrm{mg} / \mathrm{kg} /$ day followed by an 8 week recovery period. Following the observation of hair and skin pale discolorations in treated animals, $5 \mathrm{~mm}$ punch skin biopsies were collected under local anesthesia on Day 51/52 from regions of black fur on the dorsal area of all animals. The following parameters and end-points were assessed: clinical observations, ophthalmology including representative photographs of the eyelids, skin biopsies, evaluation of plasma AZD3293 concentrations, gross and microscopic pathology (including evaluation of skin biopsies and samples of plucked and clipped fur), immunohistochemistry (Melan-A and PNL-2) to identify melanocytes, and electron microscopy on skin biopsies.

\section{9-week dog study}

Six groups of Beagle dogs, each consisting of 4 males and 4 females, were given AZD3293 orally once daily for 39 weeks at dose levels of 0 (control), 1, 5 and $20 \mathrm{mg} /$ $\mathrm{kg} /$ day or AZD3293 orally once weekly for 39 weeks at dose levels of 5 or $15 / 1 \mathrm{mg} / \mathrm{kg} /$ week. Animals given $15 \mathrm{mg} / \mathrm{kg} /$ week had their dose reduced to $1 \mathrm{mg} / \mathrm{kg} /$ week on week 6 for the remainder of the main study period to increase the probability of determining the lowor no-effect dose level for the weekly dosing regimen. An additional 2 dogs/sex were given 0, 1, 5 or $20 \mathrm{mg} /$ $\mathrm{kg} /$ day once daily to assess the potential reversibility of any findings following a 13-week drug-free period. In order to identify the approximate timing of onset of microscopic hypopigmentation effects, skin biopsies were taken under local anesthesia once weekly from 2 males and 2 females per group for the first 5 weeks of treatment from the treated animals, at Weeks 11-12 from animals treated once weekly, and at Week 26 from all animals. Biopsies were taken from the region of black fur on the dorsal area. The following parameters and end-points were assessed: clinical observations, ophthalmology including representative photographs of the eyelids, skin biopsies, clinical pathology, evaluation of plasma AZD3293 concentrations, and gross and microscopic pathology (including evaluation of skin biopsies and additional staining).

\section{3-week study in older (30-32 months old) dogs}

The objective of this study was to further investigate the AZD3293-induced fur and skin pigmentation changes that were observed in the 13-week study in young dogs by studying effects of dosing in older (30-32 mos) dogs. Four groups of older Beagle dogs, each consisting of 2 males and 2 females, were dosed at levels of 0 (control), $0.1,0.3$ or $1 \mathrm{mg} / \mathrm{kg} / \mathrm{day}$ AZD3293 orally QD for 13 weeks. A further 3 groups consisting of 2 males and 2 females were dosed at levels of $0.2,1$ or $5 \mathrm{mg} / \mathrm{kg} /$ week (male dose levels reduced from 5, 15, or $25 \mathrm{mg} / \mathrm{kg} /$ week after Day 1 dosing) AZD3293 orally once weekly for 13 weeks. One group consisting of 2 males and 2 females was dosed with AZD3839, a relatively BACE1selective inhibitor, orally QD at $10 \mathrm{mg} / \mathrm{kg} /$ day for 13 weeks. Following the observation of mild hair and skin discolorations in AZD3293 treated animals, 5mm punch skin biopsies were collected under local anesthesia on Day 54/54 from regions of black fur on the dorsal area of all animals. 


\section{Study in pigmented Long-Evans rats}

The objectives of this study were to determine the potential effects on pigmented tissue, specifically eye and skin, of AZD3293 when given by daily oral gavage for 13 or 26 weeks to rats and to evaluate the potential reversibility of findings in the skin following an up to 12 -week treatment-free recovery period. Four groups of Long Evans rats (7/sex/group in Groups 1, 2, and 3 and 13 /sex/group in Group 4) were dosed at dose levels of 0 (control), 30, 100 or $200 \mathrm{mg} / \mathrm{kg} /$ day of AZD3293 once daily by oral gavage for 13 or 26 weeks. Recovery animals were euthanized following a non-dosing period of 85 days. Additional animals were included for toxicokinetic evaluation of AZD3293 during Week 13. The following parameters and endpoints were evaluated in this study: clinical signs (including fur growth), ophthalmology, plasma drug exposure, gross necropsy findings, immunohistochemistry (PNL2, Melan-A to identify melanocytes), and histopathology of the skin and eyes. Photographs were obtained at necropsy to evaluate the dose dependency and recovery of the fur and skin discoloration.

\section{Histopathology}

Tissues from each study, including skin biopsies from both dog studies, were fixed in $10 \%$ formalin, trimmed and embedded in paraffin. Sections $6 \mu \mathrm{m}$-thick were cut and stained with hematoxylin and eosin (H\&E) for histopathological evaluation. Skin samples examined were the following: in the 13-week dog studies, dorsal dark fur from Day 51-55 biopsy region, dorsal dark fur outside of the biopsy region, white fur and pigmented areas which were found to be discolored (footpad, oral mucosa, periorbital region and nose); in the 39-week dog study, dorsal dark fur area, nose and periorbital region; in the rat study, dorsal dark fur. In each study, eyes were fixed in Davidson's fixative, trimmed and embedded in paraffin. Five (for dogs) or three (for rats) levels per eye were examined using H\&E standard staining and, additionally, eyes from all animals were stained with Kinyoun's method (for lipofuscin) and regular H\&E slides were also examined under fluorescence microscopy to assess the presence of any lipofuscin. All other tissues, including all melanin-containing structures such as the Substantia Nigra, retinal pigmented epithelium, inner ear, and adrenals, were examined as part of the standard assessments associated with chronic toxicology studies.

\section{Immunohistochemistry}

Immunohistochemistry against PNL2 - a melanocyte antigen - was performed on a section consecutive to the $\mathrm{H} \& \mathrm{E}$ one in the 13-week dog studies for each skin biopsy and in the rat study for each skin sample. Sections were deparaffinized and rehydrated and antigen retrieval was performed using a Trilogy buffer solution (Cell Marque; 920P-06) and heating in microwave. Slides were then blocked using a 10\% normal goat serum for 30 minutes at room temperature and primary antibody against PNL2 (dog study: mouse monoclonal IgG1, 1:100 for 90 minutes, Santa Cruz, SC-59306; rat study: mouse monoclonal IgG (pre-diluted) for 60 minutes, Dako, N1601) was applied at room temperature. The secondary antibody was a mouse EnVision+HRP system (Dako, K4001) applied for 30 minutes at room temperature and detection was performed with a NovaRed peroxidase substrate kit from Vector (SK-4800) for 5 minutes. Sections were counterstained with Mayer's hematoxylin (30 seconds), dehydrated and mounted. Additionally, in the 13-week dog study, immunohistochemistry using an antibody directed against Melan-A (1:50 for 90 minutes at room temperature, mouse monoclonal IgG, Abcam, ab731) was also performed in order to confirm results obtained with PNL2 detection. Immunohistochemistry steps were as previously described for PNL2 staining.

\section{Electron microscopy}

In the 13-week (young) dog study, one skin biopsy per animal from a sample of control and high dose animals was cut in $1 \mathrm{~mm}$-thick sections and fixed in $4 \%$ glutaraldehyde. They were processed for electron microscopy through postfixation in $1 \%$ osmium tetroxide in $0.1 \mathrm{M}$ phosphate buffer for 4 hours, dehydrated in ethanol. Samples then transitioned through acetone, 50/50 acetone-Spurr's resin, Spurr's resin, and were embedded in Spurr's resin. Blocks were polymerized overnight at $60^{\circ} \mathrm{C}$, thick sectioned at approximately 500 $\mathrm{nm}$ and sections stained with $0.05 \%$ Toluidine Blue in $2.5 \%$ aq. sodium bicarbonate to identify areas of interest. Ultrathin sections at approximately $90 \mathrm{~nm}$ were cut from desired areas and mounted on 200 mesh copper grids. The sections were then stained with $10 \%$ uranyl acetate in methanol and Reynolds' lead citrate. Electron micrographs were taken of the grids using a Zeiss EM 900 electron microscope.

\section{Melanocyte PMEL processing assay}

The effect of BACE inhibitors on PMEL cleavage to produce $\mathrm{M} \beta$ was evaluated in human MNT1 cells (grown in RPMI with Glutamax and 10\% FCS), murine B16F10 cells (DMEM and 10\% FCS) and primary human melanocytes (Invitrogen; M254 media plus Human Melanocyte Growth Supplement). Cells were plated in 6 -well plates at a concentration of $1 \times 10^{5}$ cells/well. On the next day media was replaced, test compounds were added (prepared in DMSO; final concentration in media of $0.1 \%$ ), and cells returned to the incubator. After $24 \mathrm{hr}$ the cells were washed in Hanks Balanced Salt Solution and lysed with $250 \mathrm{uL}$ lysis reagent $(50 \mathrm{mM}$ Tris $\mathrm{pH} 7.4$, 
1\% Triton X-100, $1 \mathrm{X}$ cOmpleteTM protease inhibitor cocktail (SIGMA)). Plates were kept on ice for $15 \mathrm{~min}$ then scraped and liquid transferred to individual tubes. The tubes were incubated on ice for an additional $15 \mathrm{~min}$ with intermittent vortexing then centrifuged at 10,000 $\mathrm{x} g$ for $10 \mathrm{~min}$. Thirty micrograms of the solubilized lysate were run on NuPAGE® $10 \%$ Bis-Tris gels, electrotransferred to PVDF membrane, and then probed with aPep13h antibody (1:500; kindly provided by Prof. Michael Marks). Full length (P1) PMEL and M $\beta$ fragment were visualized using a Fast Western Super Signal Femto kit (Pierce) using conditions recommended by the vendor.

\section{In vitro assays in normal human epidermal melanocytes and reconstructed human epidermis}

All assays were run by contract at BIOalternatives (Gencay, France).

\section{Test systems used}

Cytotoxicity and melanin synthesis (monolayer cells) assays used human epidermal melanocytes (NHEM2) maintained at $37^{\circ} \mathrm{C}, 5 \% \mathrm{CO} 2$ in culture medium consisting of M254 supplemented with PMA free HMGS-2, penicillin 50, U/ml - streptomycin $50 \mu \mathrm{g} / \mathrm{ml}$, gentamicin $25 \mu \mathrm{g} / \mathrm{ml}$. Melanin synthesis (3D model) and Pmel17 labeling assays used reconstructed human epidermis (RHEm) according to the method described by Poumay, et al. $(35,36)$. Culture conditions for RHE were $37^{\circ} \mathrm{C}, 5 \% \mathrm{CO} 2$ in BIOalternatives' proprietary maintenance medium.

\section{Tyrosinase activity determination}

Isolated enzyme: tyrosinase enzyme extracted from normal human epidermal melanocytes in the assay buffer (PBS/Triton X-100). Dihydroxy-phenyl-alanine L3,4 (L-DOPA; stock solution at $10 \mathrm{mM}$ in assay buffer) was used as a test substrate.

AZD3293 or the reference (kojic acid tested at 5 concentrations from 0.125 to $1.5 \mathrm{mM}$ ) was preincubated with the enzyme for 10 minutes on ice. Then the substrate, L-DOPA ( $2 \mathrm{mM}$ final concentration) was added and the microplates were incubated at $37^{\circ} \mathrm{C}$ for 1 hour. The enzymatic activity was evaluated by measuring the optical density (OD) at $540 \mathrm{~nm}$ (Versamax microplate reader, Molecular Devices). Measurements of OD were also performed without substrate addition $(n=1)$ in order to detect potential compound interference for each test concentration. All experimental conditions were performed in $n=3$. The results were expressed as percentages of control activity and inhibition.

\section{Cytotoxicity preliminary assay}

Cell type: NHEM-2 in culture medium. Incubation time was $72+96+72$ hours. Following parameters were evaluated: MTT reduction assay and morphological observations with microscope. Briefly, following exposure to AZD3293 or positive control, the cells were incubated with MTT (tetrazolium salt) reduced in blue formazan crystals by succinate dehydrogenase (mitochondrial enzyme). After cell dissociation and formazan crystal solubilization using DMSO, the OD of the extracts at $540 \mathrm{~nm}$, proportional to the number of living cells and their metabolic activity, was recorded with a microplate reader (VERSAmax, Molecular Devices).

\section{Melanin synthesis in NDP-MSH-stimulated melanocytes (NHEM)}

Melanocytes were seeded in 24-well plates and cultured for 24 hours. The medium was then replaced by culture medium containing either AZD3293 or the reference (kojic acid, tested at $0.11,0.25$ and $0.5 \mathrm{mM}$ ) in presence of NDP-MSH (10-7 M) to stimulate melanin production. A non-stimulated control was performed in parallel. The cells were then incubated for 240 hours, with 2 treatment renewals during that period. All experimental conditions were performed in $n=3$. At the end of incubation, before performing the melanin extraction, a photo of each experimental condition was taken using an inverted microscope at x10 magnification. Melanin was then extracted by cell lysis using a $0.5 \mathrm{~N} \mathrm{NaOH}$ solution. The OD of each experimental point was measured at $405 \mathrm{~nm}$ and melanin quantity was calculated using a melanin standard curve $(0.39$ to $100 \mu \mathrm{g} / \mathrm{ml})$. The results were expressed in $\mu \mathrm{g} / \mathrm{ml}$ of melanin and in percentage of inhibition compared to stimulated control.

\section{Melanin synthesis in pigmented reconstructed human epidermis (RHEm)}

The melanocyte-containing reconstructed human epidermises (RHEm) were treated at Day 7 (D7) with AZD3293 or the reference Iklen Rucinol (applied topically at $5 \mathrm{mg} / \mathrm{cm}^{2}$ ). A non-treated control condition was performed in parallel. The RHEm were then incubated for 9 days (from D7 to D16) with one treatment renewal after 5 days of incubation (D12). All experimental conditions were performed in $n=6$ ( 5 RHEm for melanin assay and 1 RHEm for cytotoxicity assay). A control of melanin content and viability was also performed in $\mathrm{n}=6$ at the beginning of the experiment (D7). At the end of incubation, RHEm were removed from their support and melanin was extracted by adding a $90 \%$ Soluene ${ }^{\circledR}$ 350 solution (PerkinElmer). The OD of each experimental point was measured at $405 \mathrm{~nm}$ and melanin quantity was 
calculated using a melanin standard curve (0.98 to 125 $\mu \mathrm{g} / \mathrm{ml}$ ). Results were expressed in $\mu \mathrm{g} / \mathrm{ml}$ of melanin and in percentage of inhibition. To evaluate any potential cytotoxicity with drug treatment, an MTT assay was performed as described above.

\section{Pmel17 labeling in RHEm}

RHEm were treated at Day 7 (D7) with AZD3293 (applied systemically). A non-treated control condition was performed in parallel. RHEm were then incubated for 7 days (from D7 to D14) with one renewal of the treatments after 5 days of incubation (D12). All experimental conditions were performed in $n=3$. After incubation, the reconstructed human epidermal samples were snap-frozen in liquid isopentane/N2 and stored at $-80^{\circ} \mathrm{C}$.

Transversal sections were carried out using a microtome ( $5 \mu \mathrm{m}$ thickness) and the sections were fixed with an acetone/methanol mix and dried. After saturation in PBS Tween-milk 5\%, the sections were incubated with the anti-Pmel17 antibody solution. After several washes, the binding sites were revealed using a secondary antibody (GAM-Alexa 488) and the cell nuclei were stained with propidium iodide. The sections were washed and mounted between glass slides and cover slips. Digital images were recovered with fluorescent microscope (NIKON E400) at x 40 magnification. Images were captured with a NIKON DS-Ri1 and processed with NIS-Elements 3.10 software. The intensity of the fluorescent staining was measured using ImageJ software and the total number of nuclei was counted.

\section{Melanocyte dendricity assessment in a melanocytel keratinocyte co-culture}

Cell cultures used in this experiment were normal human epidermal melanocytes (NHEM; used at the 8th passage) cultured at $37^{\circ} \mathrm{C}, 5 \% \mathrm{CO} 2$; normal human epidermal keratinocytes (NHEK; used at the 3rd passage) cultured at $37^{\circ} \mathrm{C}, 5 \% \mathrm{CO} 2$;

NHEM/NHEK co-culture (2:1 ratio) cultured at $37^{\circ} \mathrm{C}$, $5 \% \mathrm{CO} 2$ in 2 volumes of supplemented KeratinocyteSFM (Epidermal Growth Factor $0.25 \mathrm{ng} / \mathrm{ml}$, pituitary extract, $25 \mu \mathrm{g} / \mathrm{ml}$, gentamycin $25 \mu \mathrm{g} / \mathrm{ml})+$ one volume of supplemented M254 medium (PMA free HMGS-2, penicillin $50 \mathrm{U} / \mathrm{ml}$, streptomycin $50 \mu \mathrm{g} / \mathrm{ml}$, gentamycin $25 \mu \mathrm{g} / \mathrm{ml}$ ). Keratinocytes and melanocytes were seeded and cultured in co-culture medium for 24 hours. The medium was then replaced with co-culture medium containing the dendricity inducer (IBMX at $200 \mu \mathrm{M}$ ) and containing or not (controls) the test compound or the reference compound vincristine $(0.03 \mu \mathrm{M})$ and the cells were incubated for 72 hours. A control with no inducer was performed in parallel (non-stimulated control). All experimental conditions were performed with $n=3$.
After incubation, culture medium was discarded and cells were rinsed, fixed and permeabilized. The cells were then labeled using a primary anti-MEL- 5 antibody protein. This primary antibody was then revealed using fluorescent secondary antibodies (GAMAlexa 488) and the cell nuclei were stained using Hoechst solution (bisbenzimide) in parallel. The acquisition of the images was performed using INCell Analyzer ${ }^{\mathrm{TM}} 1000$ (GE Healthcare). Five photos were taken per well (objective $\mathrm{x} 20$ ).

The labeling was quantified by the measurement of the dendrite surface (area in $\mu \mathrm{m}^{2}$ ) normalized to the number of melanocytes (Integration of numerical data with the Developer Toolbox 1.5, GE Healthcare software). Selected representative images of the observed effects are presented in Figure 6. The inter-group comparisons were performed by an unpaired Student's t-test.

\section{Human skin biopsies}

A multiple ascending dose study (NCT01795339) was conducted to evaluate the safety, tolerability, pharmacokinetics, and pharmacodynamics of doses of 15 or $50 \mathrm{mg}$ AZD3293 once daily (QD) for 12 consecutive days or $70 \mathrm{mg}$ once weekly $(\mathrm{QW})$ vs. matching placebos in elderly subjects (Part $1, \mathrm{n}=31$ ), and 15,50 , or $150 \mathrm{mg}$ AZD3293 QD or matching placebos for 12 consecutive days in patients with mild to moderate Alzheimer's disease (Part 2, n=16) $(37,38)$. Full skin biopsies were obtained from the gluteal region (sun-protected area) of the 16 study subjects in Part 2 of the study, before and after daily treatment with AZD3293 (15 mg, $50 \mathrm{mg}$ or 150 $\mathrm{mg}$ ) or placebo. Subjects in Part 2 were mostly female $(68 \%)$, mostly Caucasian or Asian (44\% each), and had a mean age of 64 years and a mean body mass index of 25.8. Histological slides were prepared and stained with hematoxylin-eosin, for general examination of skin morphology, and with Fontana-Masson, to visualize the melanocytes and the melanin content in keratinocytes. Melanin content was then scored in Stratum basale (Sb) and Stratum spinosum (Ss) semi-quantitatively according to the following scheme: for $\mathrm{Sb}, 0-$ none/negligible amounts, $1-$ moderate/strong stain in $<5 \%$ of cells, weak stain in $<50 \%$ of cells, continuous staining, 2 - moderate/ strong stain in $5-20 \%$ of cells, weak in $>50 \%$ of cells, discontinuous staining, 3 - moderate/strong stain in $20-50 \%$ of cells, all cells positive, continuous staining, $4-$ moderate/strong stain in $>50 \%$ of cells, all cells positive, continuous staining; for Ss, 0 - none/negligible amounts, 1 - stain in $<10 \%$ of cells, 2 - stain in $10-30 \%$ of cells, 3 - stain in $30-60 \%$ of cells, 4 - stain in $>60 \%$ of cells. Examples of staining grades in $\mathrm{Sb}$ and $\mathrm{Ss}$ are shown in Figure 7. Melanin staining in hair follicles was also registered, although hair follicles were only present in a minority of the biopsies. The scoring pathologist was blinded with respect to individual identity, skin type, treatment, and whether the sample was pre- or post- 
dose. After scoring, subjects were stratified according to treatment, patient ID and pre- or post-dose sampling.

Figure 1. Photographs of homogenous hypopigmentation of nose, periorbital skin and dark fur following 12 weeks of treatment in a 39-week toxicology study in young Beagle dogs treated with vehicle (control, top panel), AZD3293 $20 \mathrm{mg} / \mathrm{kg} /$ day (middle panel), or AZD3293 $5 \mathrm{mg} / \mathrm{kg} /$ week (bottom panel). Note the striped-pattern appearance of the eyebrows and whiskers in the weekly dosing group (see inset photo), indicating acute functional recovery of normal pigmentation in the interval between the weekly doses of AZD3293. Dorsal dark fur area photographs are taken from the areas previously shaven for skin biopsies. The tan colored fur did not appreciably change in appearance

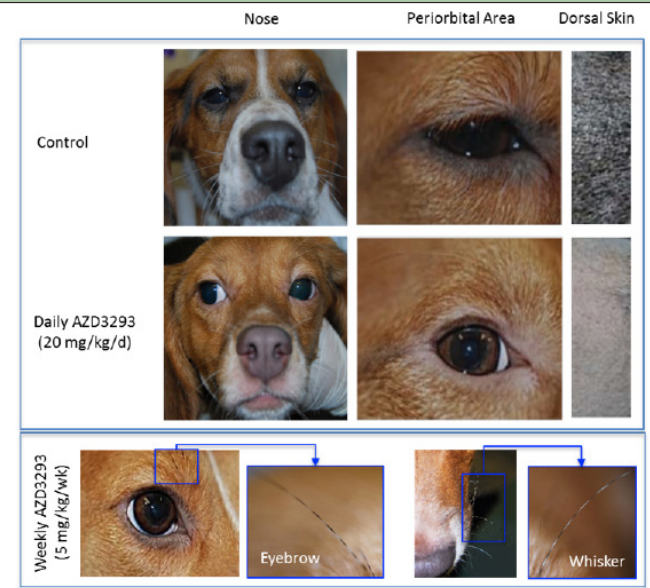

Results

\section{Depigmentation of skin, oral mucosa, and dark fur in dogs}

\section{3-week toxicology study in young Beagle dogs}

In the 13-week dog toxicology study, 8-10 months old Beagle dogs (at the start of dosing; a typical age for Beagle dogs used in regulatory toxicology studies) treated with AZD3293 displayed gradually diminishing pigmentation of the visible pigmented skin (around the eyes and the nose), black-colored fur, mucosa inside the oral cavity, and paw pads, which was first noticed from the 5th-6th week of dosing (Figure $1)$. The hypopigmentation, which was confluent and homogeneous, was initially noticed to affect the eyelid margins and muzzle. By the end of the 6th week of dosing, however, the hypopigmentation effect was also observed on the plantar surfaces of the paws, the hard palate, and the mucosa-skin border on the lips. Slight hypopigmentation of the black fur was also observed at that time. By the end of the 13-week dosing period, all dogs treated with AZD3293 showed pronounced hypopigmentation of skin and fur, with no apparent dose relationship in severity.

Figure 2. Top panel. Histopathology micrographs of hair follicle cross-sections in skin biopsies taken following 7 weeks of treatment in the 13-week toxicology study in young Beagle dogs treated with vehicle (control, left column), daily doses of AZD3293 (middle column), and after 12 weeks of treatment-free recovery (right column) utilizing $\mathrm{H} / \mathrm{E}$ staining and/ or PNL2 immunohistochemistry methodologies to visualize melanin and melanocyte morphology, and for qualitative assessment of melanocyte numbers in the hair follicles. Arrow indicates the location of the hair follicle and the bracket indicates the melanocyte zone surrounding the hair shaft within the follicle. Bottom panel. Shown are representative electron microscopy images of hair follicle melanocytes from skin biopsies taken following 7 weeks of treatment in the 13-week toxicology study in young Beagle dogs treated with vehicle (control, left panel) or daily doses of AZD3293 (right panel). Note the more numerous melanosomes and their variable shapes and sizes in the AZD3293 treatment group.

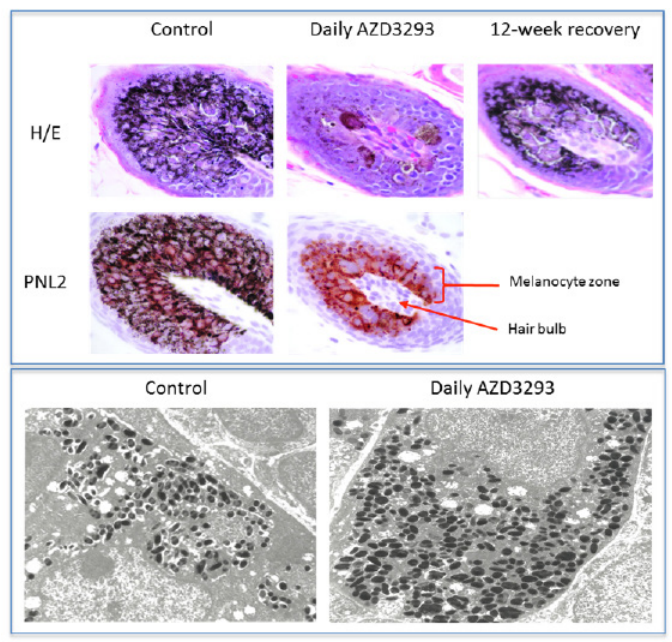

Full skin biopsies from the backs were obtained at the end of Week 8 of dosing for histopathological, immunohistochemical, and electron microscopy methods to define the hypopigmentation effect at the cellular level. There were no melanocytes or melanin granules detected in the epidermis itself in any of the biopsy samples, including the controls, indicating that epidermis underneath the fur in this location is normally not pigmented. The hair follicles were the only structures in these biopsies that contained melanin and in which melanocytes could be detected. A marked decrease in pigmentation was noted in the hair shaft and hair follicles of all treated animals (Figure 2, top panel). This finding was usually associated with the presence of abnormal-looking cells in the hair follicle, around the dermal papilla. These cells contained variable amounts of light-to-dark brown pigment (likely melanin) and were 
occasionally hypertrophied and/or vacuolated. There was, however, no evidence of inflammation, cell death or fibrosis. The extent of PNL2- or Melan A-positive staining of cells within the hair follicles was generally comparable between controls and treated animals (Figure 2 , top panel) meaning the overall number of melanocytes appeared to not be affected in treated animals. Individual melanocytes were often well delineated, and their staining intensity appeared to be similar between control and treated animals. Additionally, the hypertrophied/ vacuolated cells observed in H\&E sections stained positive with PNL-2 and Melan-A and were therefore identified as abnormal melanocytes.

Figure 3. Melanin content throughout the skin layers visualized via $H / E$ staining in terminal nose skin samples from young Beagle dogs following 13 weeks of treatment with vehicle (control, panels A and C) or daily doses of AZD3293 (panels B and D). Note the pronounced melanization (darker appearance) of the melanocyte cell layer and the deepest layers of keratinocytes in the control samples and the near complete absence of melanization in the representative sample from a dog treated daily with AZD3293. Photographs in panels A and B are taken at low magnification, panels $C$ and $D$ show same fields of vision at higher magnification.
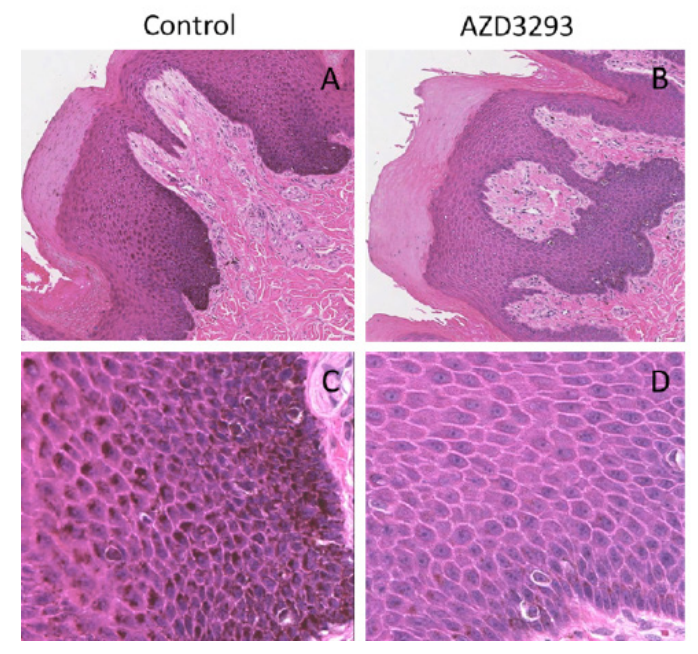

The electron microscopy analysis of the dog biopsy material (Figure 2, bottom panel) largely confirmed the histopathology results, indicating that hair follicle melanocytes were filled with melanin-containing formations, likely melanosomes that differed in size and shape from the control (the melanosomes from AZD3293treated animals were more round in shape and typically bigger). These observations are consistent with alterations in melanocyte morphology and changes in melanosome size and shape seen in PMEL-deficient mice $(32,39)$.

At the end of the dosing period, no qualitatively new findings were revealed in necropsy samples as compared to the punch skin biopsies obtained after 8 weeks of treatment. In the non-haired skin areas, such as the muzzle, the melanin content in the epidermal keratinocytes of AZD3293-treated animals was dramatically lower than in the controls (Figure 3). While some of the cells on the basal membrane, presumably melanocytes, still contained some degree of melanization (Figure 3D), keratinocytes in all layers were almost completely devoid of melanin. The rare melanocytes observed usually had an abnormal hypertrophic appearance but this feature was less pronounced compared to hair follicle melanocytes. In addition to skin and mucosa, care was taken to carefully examine other melanin-containing tissues such as certain brain substructures (i.e., Substantia Nigra), retinal pigmented epithelium, inner ear, and adrenals. It was confirmed that apart from skin, oral mucosa and hair follicles, no other pigmented tissues showed any signs of depigmentation or any other cellular/ morphological changes.

By the end of the 8-week recovery period, there was nearly full recovery of pigmentation at the cellular level (normal melanin content and appearance of melanocytes and keratinocytes in both epidermis and hair follicles; Figure 2, top panel) as well as partial recovery of the appearance of skin and fur, indicating that the hypopigmentation effects were reversible.

\section{9-week toxicology study in young Beagle dogs}

In the 39-week toxicology study with young Beagle dogs, starting at Week 5 of AZD3293 treatment, pale skin was observed at all dose levels in the eyelids and around the nose and muzzle. These changes were later accompanied by the appearance of fading pigmentation of the dark fur (starting at Week 21), but not the tancolored fur (Table 1).

Serial weekly skin biopsies collected in this study allowed an assessment of the time-course of histopathological changes. After 2 weeks of treatment, moderate to marked decreases in pigmentation of the hair follicle was noted in all interim skin biopsies from regions of black fur in all animals treated with AZD3293 daily or weekly (Table 2). During the first week, decreased pigmentation was only noted in the hair follicle, and this change extended to large portions of the hair shaft in the following weeks. The severity of this change increased for the first three weeks and then stabilized, except for animals given $15 / 1 \mathrm{mg} / \mathrm{kg} /$ week in which the severity started to decrease at Week 11/12, suggesting partial recovery following the decrease in dose level. Starting at Week 4, rare to few abnormal pigmented cells were seen in affected hair follicles and were characterized as enlarged cells variably filled with melanin pigment.

In dogs treated daily with AZD3293, there was a slight increase in severity at $\geq 5 \mathrm{mg} / \mathrm{kg} /$ day compared to 1 $\mathrm{mg} / \mathrm{kg} /$ day during the first week, but this difference in severity was no longer obvious from week 2 onwards.

In dogs treated weekly with AZD3293, an interesting 
Table 1. Clinical appearance of young Beagle dogs following 39 weeks of daily treatment with various doses of AZD3293 or vehicle (control). Shown are number of animals with pigment-related findings within each sex

\begin{tabular}{|c|c|c|c|c|c|c|c|c|c|c|c|c|}
\hline Dosing Frequency & \multicolumn{8}{|c|}{ Daily } & \multicolumn{4}{|c|}{ Weekly } \\
\hline Dose (mg/kg/day) & \multicolumn{2}{|c|}{ Control } & \multicolumn{2}{|c|}{1} & \multicolumn{2}{|c|}{5} & \multicolumn{2}{|c|}{20} & \multicolumn{2}{|c|}{5} & \multicolumn{2}{|c|}{$15 / 1^{a}$} \\
\hline $\mathbf{N}$ & \multicolumn{2}{|c|}{8} & \multicolumn{2}{|c|}{8} & \multicolumn{2}{|c|}{8} & \multicolumn{2}{|c|}{8} & \multicolumn{2}{|c|}{8} & \multicolumn{2}{|c|}{8} \\
\hline Sex & $\mathbf{M}$ & $\mathbf{F}$ & $\mathbf{M}$ & $\mathbf{F}$ & $\mathbf{M}$ & $\mathbf{F}$ & $\mathbf{M}$ & F & $\mathbf{M}$ & $\mathbf{F}$ & $\mathbf{M}$ & $\mathbf{F}$ \\
\hline Discoloration of pigmented fur & - & - & 4 & 4 & 4 & 4 & 4 & 4 & 3 & 2 & - & 1 \\
\hline Discoloration of dark dorsal fur & - & - & 4 & 4 & 4 & 4 & 4 & 4 & 3 & 3 & 1 & 1 \\
\hline Discoloration of skin on nose & - & - & 4 & 4 & 4 & 4 & 4 & 4 & 3 & 2 & - & 1 \\
\hline Discoloration of skin in periorbital area & - & - & 4 & 4 & 4 & 4 & 4 & 4 & 3 & 4 & 2 & 1 \\
\hline
\end{tabular}

a. Animals were initially dosed at $15 \mathrm{mg} / \mathrm{kg} /$ day for 3 or 4 weeks, after which their dose was reduced to $1 \mathrm{mg} / \mathrm{kg} / \mathrm{day}$.

Table 2. Histopathology results from skin biopsies following 2 weeks of treatment with daily or weekly doses of AZD3293 or vehicle (control) in the 39-week chronic toxicology study in young Beagle dogs. Shown are number of animals with pigment-related findings by severity within each sex

\begin{tabular}{|c|c|c|c|c|c|c|c|c|c|c|c|c|}
\hline Dosing Frequency & \multicolumn{8}{|c|}{ Daily } & \multicolumn{4}{|c|}{ Weekly } \\
\hline Dose (mg/kg/day) & \multicolumn{2}{|c|}{ Control } & \multicolumn{2}{|c|}{1} & \multicolumn{2}{|c|}{5} & \multicolumn{2}{|c|}{20} & 5 & & \multicolumn{2}{|c|}{$15 / 1^{a}$} \\
\hline $\mathbf{N}$ & \multicolumn{2}{|c|}{4} & \multicolumn{2}{|c|}{4} & \multicolumn{2}{|c|}{4} & \multicolumn{2}{|c|}{4} & 2 & & \multicolumn{2}{|c|}{2} \\
\hline Sex & $\mathbf{M}$ & F & $\mathbf{M}$ & $\mathbf{F}$ & $\mathbf{M}$ & F & $\mathbf{M}$ & F & $\mathbf{M}$ & F & $\mathbf{M}$ & $\mathbf{F}$ \\
\hline \multicolumn{13}{|c|}{ Decreased pigmentation of hair follicle } \\
\hline $\mathrm{N}$ & 2 & 2 & $1^{\mathrm{b}}$ & 2 & $1^{\mathrm{b}}$ & 2 & $1^{\mathrm{b}}$ & 2 & $0^{\mathrm{b}}$ & 1 & 1 & 1 \\
\hline Minimal & - & - & - & - & - & - & - & - & - & - & - & - \\
\hline Mild & - & - & - & - & - & - & - & - & - & - & - & - \\
\hline Moderate & - & - & 1 & - & - & - & - & - & - & 1 & - & 1 \\
\hline Marked & - & - & - & 2 & 1 & 2 & 1 & 2 & - & - & 1 & - \\
\hline
\end{tabular}

a. Animals were initially dosed at $15 \mathrm{mg} / \mathrm{kg} /$ day for 3 or 4 weeks, after which their dose was reduced to $1 \mathrm{mg} / \mathrm{kg} /$ day; b. Section(s) obtained for 1 animal in each of these groups did not contain a hair follicle.

Table 3. Clinical appearance of older Beagle dogs following 13 weeks of daily treatment with various doses of AZD3293, $10 \mathrm{mg} / \mathrm{kg} /$ day AZD3839, or vehicle (control). Shown are number of animals with pigment-related findings by severity within each sex

\begin{tabular}{|c|c|c|c|c|c|c|c|c|c|c|}
\hline Treatment & \multicolumn{8}{|c|}{ AZD3293 } & \multirow{2}{*}{\multicolumn{2}{|c|}{$\begin{array}{c}\text { AZD3839 } \\
10\end{array}$}} \\
\hline Dose (mg/kg/day) & \multicolumn{2}{|c|}{ Control } & \multicolumn{2}{|c|}{0.1} & \multicolumn{2}{|c|}{0.3} & \multicolumn{2}{|c|}{1} & & \\
\hline $\mathbf{N}$ & \multicolumn{2}{|c|}{4} & \multicolumn{2}{|c|}{4} & \multicolumn{2}{|c|}{4} & \multicolumn{2}{|c|}{4} & \multicolumn{2}{|c|}{4} \\
\hline \multicolumn{11}{|c|}{ Discoloration of dark dorsal fur } \\
\hline $\mathrm{N}$ & 2 & 2 & 2 & 1 & 2 & 2 & 2 & 2 & 2 & 2 \\
\hline Marked & - & - & - & - & 1 & - & 2 & 1 & - & - \\
\hline \multicolumn{11}{|c|}{ Discoloration of skin on nose } \\
\hline $\mathrm{N}$ & 2 & 2 & 2 & 2 & 2 & 2 & 2 & 2 & 2 & 2 \\
\hline Minimal & - & - & 1 & 1 & - & 2 & 1 & - & - & - \\
\hline $\mathrm{N}$ & 2 & 2 & 2 & 2 & 2 & 2 & 2 & 2 & 2 & 2 \\
\hline Minimal & - & - & 1 & 1 & 2 & 1 & - & - & - & - \\
\hline Mild & - & - & - & 1 & - & 1 & 2 & - & - & - \\
\hline Moderate & - & - & - & - & - & - & - & 2 & - & - \\
\hline
\end{tabular}


striped-pattern appearance (Figure 1, bottom panel) was observed on some of the facial hairs in the eyebrow and whisker areas. The growth of these facial hairs differ from the rest of the Beagle fur by having a substantially longer anagen phase (similar to that of human scalp hair follicles), in which there is continuous melanin production and hair growth for up to 5 years. The white spaces on the otherwise black hair represent the transient disruption in melanin production caused by weekly treatment with AZD3293 (plasma $\mathrm{t}_{1 / 2}$ of $7.4 \mathrm{hr}$ ). Based on the plasma concentrations in the terminal phase, we estimate that after each weekly dose, free plasma concentrations would drop below $0.9 \mathrm{nM}$, the BACE2 IC50, within 3-4 days of dosing. This result combined with the fact that the pigmentation of hair shaft was seamlessly restored prior to administration of each weekly dose suggests rapid functional recovery of melanin production following compound washout.

Figure 4. Shown are representative photographs of AZD3293-induced dose-dependent depigmentation in Long-Evans rats following 26 weeks (6 months) of daily dosing (left panel), and appearance of representative high dose group ( $200 \mathrm{mg} / \mathrm{kg} /$ day) male and female rats after 12 weeks of drug-free recovery period (right panel). Note the partial recovery of hair pigmentation particularly prominent in the male rats, signified by the motley appearance of the dark fur

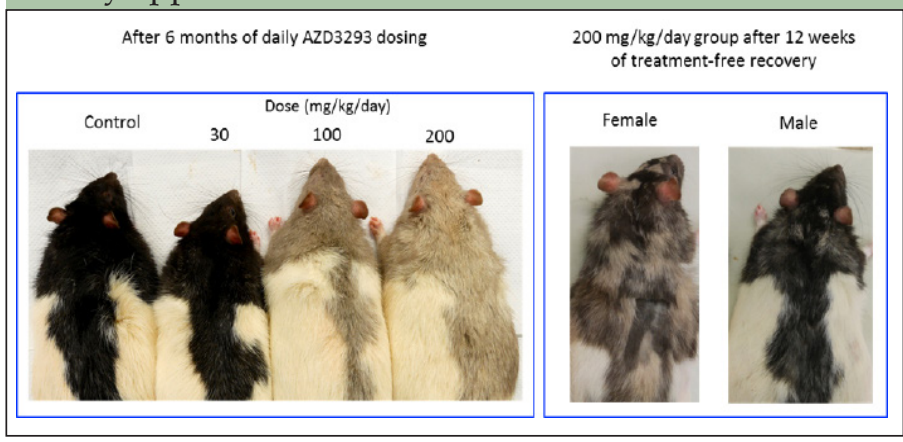

At necropsy, minimal to severe decreased pigmentation of the black fur areas was observed in all AZD3293-treated animals in at least one of the sections examined, except in one male dog given $15 / 1 \mathrm{mg} / \mathrm{kg} /$ week in which all sections were histologically normal. Decreased pigmentation was more pronounced in animals treated daily compared to weekly (as illustrated in Figure 1). In animals treated daily, the severity slightly increased with the dose, and in animals treated weekly, the severity was slightly higher at $5 \mathrm{mg} / \mathrm{kg} /$ week compared to $15 / 1 \mathrm{mg} / \mathrm{kg} /$ week. The decreased pigmentation observed microscopically correlated with the pale discoloration noted at necropsy.

As was seen in the 13-week study, no other pigmented tissues showed any signs of depigmentation or any other cellular/ morphological changes and a clear trend towards recovery of macroscopic changes was observed by the end of the 8-week treatment-free recovery period.

\section{Effects of AZD3293 and AZD3839 in older Beagle dogs}

Daily dosing of AZD3293 at 0.3 and $1 \mathrm{mg} / \mathrm{kg} /$ day resulted in the hair, particularly in the areas previously shaven over the biopsy sites, being noted as grey in color from Day 78 and 80 of the study onwards in females and males, respectively (Table 3 ). By the end of the dosing period, the dark portions of the dog's coats were generally more grey in color, their noses were pale and the eyebrows of one male and one female were white with a black tip and the muzzle whiskers of the male dog were also white with black tips. In contrast, daily dosing of AZD3293 at $0.1 \mathrm{mg} / \mathrm{kg} /$ day by the end of the dosing period resulted in only 2 out of 4 dogs developing minimal to mild depigmentation of skin, and 1 out of 4 dogs developed mild discoloration of dark dorsal fur at this dose level.

Figure 5. Representative Western blot analysis of Human MNT1 cells incubated for $24 \mathrm{hr}$ with either DMSO solution or different concentrations (as indicated below the blot) of three unique BACE1 inhibitor compounds with differing affinities for BACE2 and then processed to identify parent (P1) and proteolytically cleaved $\mathrm{M} \beta$ fragment. Vertical black and blue lines represent the IC50 values for each compound for inhibition of human BACE1 and BACE2 enzymes, respectively (AZD3293: 0.6 and 0.9 $\mathrm{nM}$; AZD3839: 36 and $444 \mathrm{nM}$; and AZ1340: 308 and >5,000 nM). Bolded arrows on the right side indicate the approximate expected molecular weights of $\mathrm{P} 1$ and $\mathrm{M} \beta$ fragment, respectively

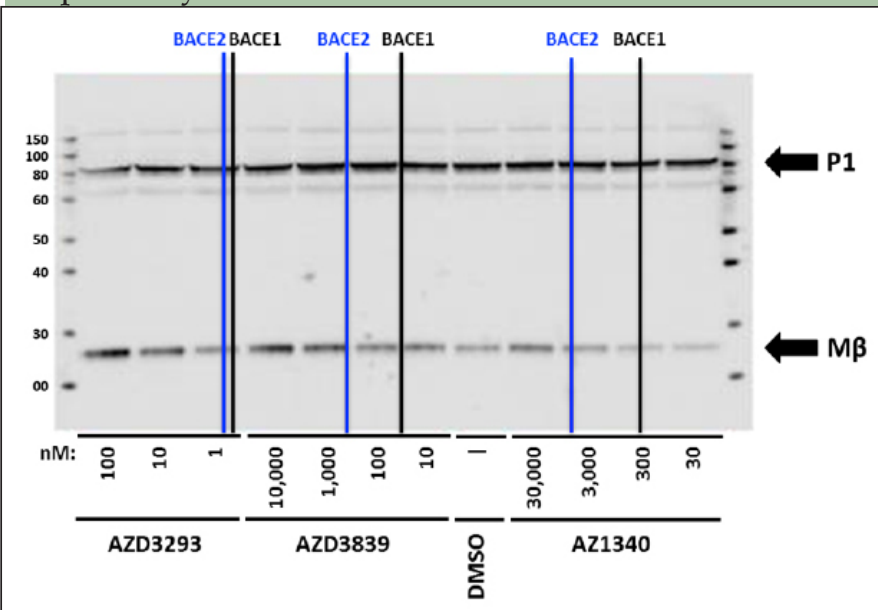

P1 = premelanosome protein; $M \beta=$ fragment of PMEL that contains a disulfide-linked transmembrane domain; $\mathrm{nM}$ = nanomolar; DMSO = dimethyl sulfoxide.

Weekly dosing of AZD3293 at 1 and $5 \mathrm{mg} / \mathrm{kg} /$ week produced changes similar to what was seen in animals dosed daily with one exception: a black and white striped-pattern appearance was noted in the eyebrows of one male and both females and muzzle whiskers in one male, as seen in the younger dogs on dosed weekly. Weekly dosing of AZD3293 at $0.2 \mathrm{mg} / \mathrm{kg} /$ week had only 
Table 4. Lowest observed effect levels (LOELs) for hypopigmentation in Beagle dogs and Long-Evans rats with corresponding mean maximum plasma free (unbound) AZD3293 concentrations (Free Cmax), fold over the 50\% inhibitory concentration (IC50) of AZD3293 for the human BACE2 enzyme (0.9 nM), and fold over the mean maximum plasma free AZD3293 values observed at steady-state after daily dosing in humans of $15 \mathrm{mg}(14.4 \mathrm{nM})$ and $50 \mathrm{mg}(61.5$ nM) AZD3293

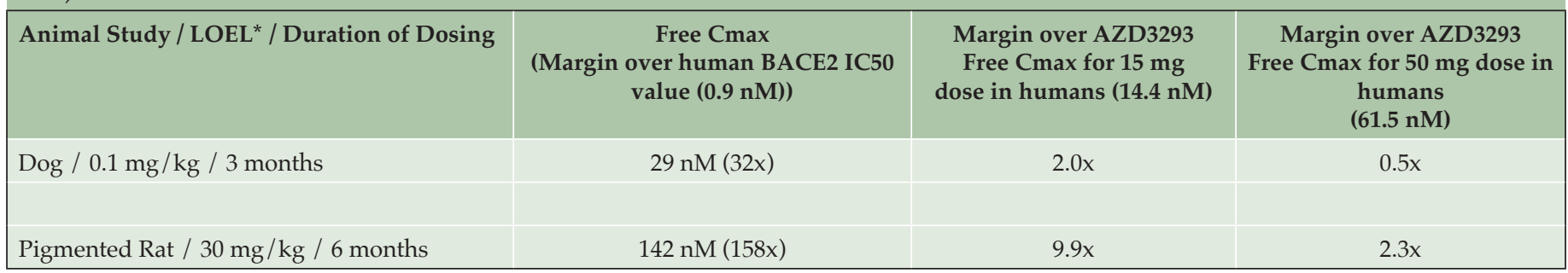

${ }^{*}$ LOEL = Lowest observed effect level; corresponds to minimal changes in histopathology and clinical appearance of AZD3293-treated animals

Table 5. Clinical appearance of animals after 13 and 26 weeks of daily dosing or after 12 weeks of recovery following dosing with various doses of AZD3293 or vehicle (control) in Long-Evans rats. Shown are number of animals with corresponding findings within each sex and observation week.

\begin{tabular}{|c|c|c|c|c|c|c|c|c|c|c|c|c|}
\hline \multirow{2}{*}{$\begin{array}{l}\text { Dose (mg/kg/day) } \\
\text { Phase }\end{array}$} & \multicolumn{3}{|c|}{ Control } & \multicolumn{3}{|c|}{30} & \multicolumn{3}{|c|}{100} & \multicolumn{3}{|c|}{200} \\
\hline & $\begin{array}{l}\text { Treat- } \\
\text { ment }\end{array}$ & & Recovery & Treatment & & Recovery & Treatment & & Recovery & Treatment & & Recovery \\
\hline Week & 13 & 26 & +12 & 13 & 26 & +12 & 13 & 26 & +12 & 13 & 26 & +12 \\
\hline N (Males/Females) & $3 / 3$ & $2 / 2$ & $2 / 2$ & $3 / 3$ & $2 / 2$ & $2 / 2$ & $3 / 3$ & $2 / 2$ & $2 / 2$ & $3 / 3$ & $2 / 2$ & $3 / 4$ \\
\hline Pale fur discoloration & $-/-$ & $-/-$ & $-/-$ & $3 / 3$ & $2 / 2$ & $-/-$ & $3 / 3$ & $2 / 2$ & $-/-$ & $3 / 3$ & $2 / 2$ & $-/-$ \\
\hline $\begin{array}{l}\text { Mottled fur discoloration } \\
\text { following recovery }\end{array}$ & $-/-$ & $-/-$ & $-/-$ & $-/-$ & $-1-$ & $-1-$ & $-1-$ & $-1-$ & $2 / 2$ & $-/-$ & $-/-$ & $3 / 4$ \\
\hline
\end{tabular}

minimal effects at the end of the dosing period resulting in a pale nose in one female and the muzzle whisker in a male showing the striped-pattern appearance.

Histopathological examination of skin biopsies taken on days 54/55 showed that AZD3293 produced minimal to moderate decreases in hair pigmentation in females at $\geq 0.1 \mathrm{mg} / \mathrm{kg} /$ day, in males at $\geq 0.3 \mathrm{mg} / \mathrm{kg} /$ day, and in both sexes at $5 \mathrm{mg} / \mathrm{kg} /$ week. This change was usually restricted to hair follicles but was sometimes extended into a few hair shafts on a given section. It was characterized by a decreased pigment content in the follicle, sometimes associated with the presence of abnormally pigmented cells that were hypertrophic and contained a variable amount of light to dark brown granular pigment (likely melanin). Such abnormal cells were noted in 4 animals at $\geq 0.3 \mathrm{mg} / \mathrm{kg} /$ day. These findings were of a higher severity grade in animals given $1 \mathrm{mg} / \mathrm{kg} /$ day. There were no treatment-related histopathological findings attributed to administration of the BACE1-selective compound AZD3839.

At the end of the 13-week treatment period with AZD3293, microscopic findings in the skin and/or hair follicles (in dark fur areas, nose, and periorbital area) were seen in both sexes at $\geq 0.1 \mathrm{mg} / \mathrm{kg} /$ day and $\geq 1 \mathrm{mg} /$ $\mathrm{kg} /$ week. These changes had generally increased in incidence and severity compared to biopsies collected on days 54/55. Thus, all pigmented areas that were examined (dorsal dark fur, nose, and periorbital area) had minimally to markedly decreased pigmentation. In haired skin, decreased pigmentation was usually graded moderate or marked, was diffuse in the hair follicle, and usually extended into hair shafts. A few abnormal (hypertrophied/vacuolated) pigmented cells were present in hair follicles, around the dermal papilla in most daily treated animals $(9 / 12)$ vs. rare $(3 / 12)$ in weekly treated dogs. These cells were identified as melanocytes with immunohistochemistry. Minimally to moderately decreased pigmentation was noted in nonhaired skin epidermis (nose and periorbital region).

Daily dosing of AZD3839 (12-fold selective for BACE1) at $10 \mathrm{mg} / \mathrm{kg} /$ day achieved a maximum unbound plasma drug exposure $(490 \mathrm{nM})$ that was 14-fold over its BACE1 IC50 and approximately equal to its BACE2 IC50, and resulted in no treatment-related macroscopic or histopathological findings after 13 weeks of treatment.

To summarize the dog studies, AZD3293 produced a pronounced dose-dependent hypopigmentation of pigmented skin, mucosa, and fur when administered chronically to dogs. Histopathological changes in the hair follicles were not observed until after at least 1 week of daily dosing and the first visible signs of hypopigmentation did not appear until after 3-5 weeks of daily dosing. The appearance of the animals correlated with the marked decrease of melanin in non-haired epidermal and hair follicle keratinocytes and changes in the morphological appearance of melanocytes. The effects were less severe with weekly as compared to daily dosing; effects appeared sooner and were more pronounced in younger animals as compared to older ones. The histopathological changes in the hair 
follicle and epidermis had fully recovered by the end of the 8-week or 13-week treatment-free recovery periods following the 13 and 39 weeks of dosing in dogs, respectively. There was also partial recovery of pigmentation of the skin and fur by the end of the corresponding recovery periods.

The lowest plasma drug exposure at which hypopigmentation was detected in the dog studies $(0.1 \mathrm{mg} / \mathrm{kg}$, low-dose group in the 13-week study in older dogs) was about 3-fold below the mean plasma drug exposure achieved at steady-state in the multiple ascending dose human study at the $150 \mathrm{mg} /$ day dose (data not shown) and approximately half of the mean plasma drug exposure at the $50 \mathrm{mg} /$ day dose in that study (Table 4). Skin biopsy results from this study are described below. The $50 \mathrm{mg} /$ day dose level is the highest dose level being tested in the ongoing Phase 3 studies of AZD3293 in Alzheimer's disease (NCT02245737 and NCT02783573).

Figure 6. (A) AZD3293 (1 $\mu \mathrm{M}$ for 10 days; middle panel) had no effects on melanocyte viability, appearance, and melanin production (inset numbers are the percentage of control melanin levels) relative to control (left panel) in cultures of normal human melanocytes stimulated with NDP-MSH. Kojic acid ( $250 \mu \mathrm{M}$ for 10 days, right panel) was used as a positive control and it inhibited melanin production by $86 \%$. (B) AZD3293 had no effect on melanocyte viability and/or dendricity following 10 days of incubation ( $10 \mu \mathrm{M}$; right panel) relative to control (left panel) in co-cultures of primary human melanocytes and keratinocytes

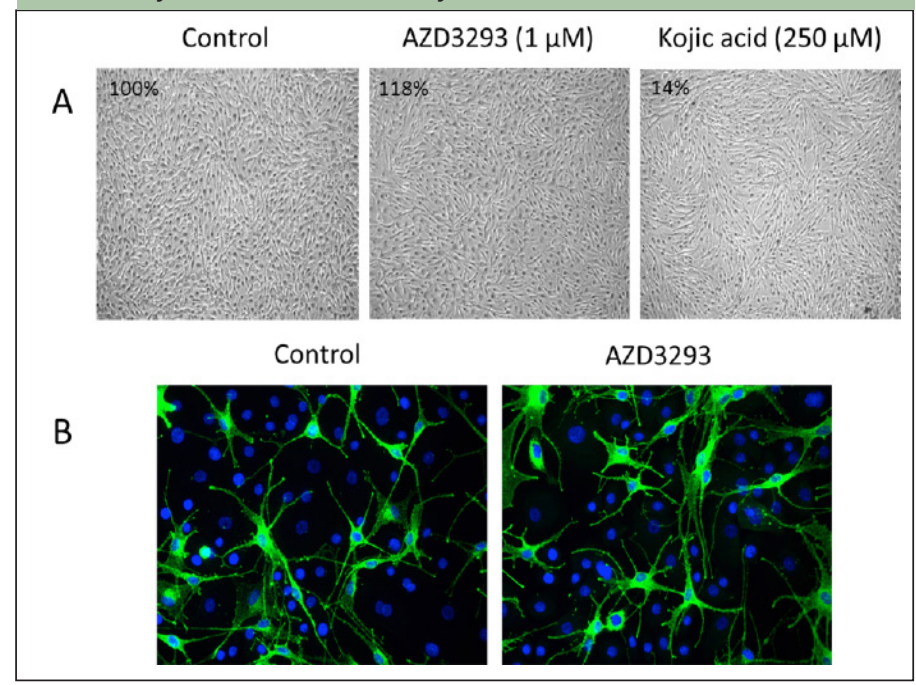

\section{Effects in pigmented Long-Evans rats}

At the end of the 6-month treatment period, AZD3293related pale homogeneous discoloration in the pigmented fur was observed in the skin of all treated animals (Figure 4). The appearance of the pigmented fur was mottled during the first few months of the study as the effect developed gradually over the pigmented area in accordance with the natural hair cycle in the rat. Fur depigmentation correlated microscopically with decreased hair follicle pigmentation seen at $\geq 30 \mathrm{mg} / \mathrm{kg} /$ day. Fur growth or re-growth per se did not seem to be affected by AZD3293 treatment.

Figure 7. Top panel. Examples of Grade 1 (left panel) and Grade 4 (right panel) melanization level scoring in Fontana-Masson stained (to visualize melanin in melanocytes and keratinocyte layers) paraffin-embedded sections from skin biopsies obtained from Alzheimer's disease subjects treated with 13 doses of AZD3293 over 14 days at levels of up to $150 \mathrm{mg}$. Bottom panel. Scores quantifying change from baseline in skin melanization in two epidermal layers (stratum basale and stratum spinosum) are summarized in the accompanying data grid showing decreased (-1), no change (0), or increased $(+1)$ melanin content for skin biopsies from human subjects receiving placebo, $15 \mathrm{mg}$, $50 \mathrm{mg}$, or $150 \mathrm{mg}$ AZD3293 over 14 days

\begin{tabular}{|c|c|c|c|c|c|c|c|c|}
\hline Gra & $1 \mathrm{~m}$ & lanizat & & & Grac & e $4 \mathrm{~m}$ & anizati & \\
\hline \multirow{2}{*}{$\begin{array}{l}\text { Melanin } \\
\text { Change }\end{array}$} & \multicolumn{4}{|c|}{ Stratum basale } & \multicolumn{4}{|c|}{ Stratum spinosum } \\
\hline & PBO & $\begin{array}{c}15 \mathrm{mg} \\
\text { AZD3293 }\end{array}$ & $\begin{array}{r}50 \mathrm{mg} \\
\text { AZD3293 }\end{array}$ & $\begin{array}{l}150 \mathrm{mg} \\
\text { AZD } 3293\end{array}$ & PBO & $\begin{array}{r}15 \mathrm{mg} \\
\text { AZD } 299\end{array}$ & $\begin{array}{r}50 \mathrm{mg} \\
\text { AZD } 3293\end{array}$ & $\begin{array}{l}150 \mathrm{mg} \\
\text { AZD } 293\end{array}$ \\
\hline-1 & 2 & 1 & 0 & 1 & 0 & 0 & 0 & 1 \\
\hline 0 & 2 & 3 & 3 & 2 & 4 & 1 & 4 & 3 \\
\hline+1 & 0 & 0 & 1 & 1 & 0 & 3 & 0 & 0 \\
\hline
\end{tabular}

After a 12-week treatment-free recovery period, mottled discoloration of the pigmented fur was observed in all animals previously given $\geq 100 \mathrm{mg} / \mathrm{kg} /$ day, indicative of partial recovery of fur pigmentation (Table 5). Compared to female rats, male rats at $\geq 100$ $\mathrm{mg} / \mathrm{kg} /$ day showed somewhat faster recovery to the original tonality of the dark fur (Figure 4). At the end of the 12-week recovery period in the $30 \mathrm{mg} / \mathrm{kg} /$ day group homogenous dark fur was observed in all animals indicating complete recovery of fur pigmentation at this dose level by the 12-week time point.

H\&E stained skin sections showed minimally to severely decreased hair follicle pigmentation at $\geq 30 \mathrm{mg} /$ $\mathrm{kg}$ / day with an increased incidence and severity at $\geq 100$ $\mathrm{mg} / \mathrm{kg} /$ day (data not shown). At $\geq 100 \mathrm{mg} / \mathrm{kg} /$ day, the decreased pigmentation was present in the hair follicle and extended into the hair shaft; it was graded moderate to severe based on the amount of pigment present in a given section (i.e. some of the variability observed within a group was likely due to differences in the number of hair follicles present in a section). At $30 \mathrm{mg} / \mathrm{kg} /$ day, decreased pigmentation was observed as a decreased amount of pigment present in individual cells of some 
hair follicles in a section and was graded minimal. Additionally, at $\geq 100 \mathrm{mg} / \mathrm{kg} /$ day, rare abnormal pigmented cells (increased in size and filled with variable amount of light- to dark-brown pigment) were present in the hair follicles of a few animals.

After 4 weeks of recovery there were no AZD3293related effects on hair follicles in males, which was suggestive of complete recovery of the microscopic findings in this gender. The absence of hair follicles the in skin sections obtained from females prevented evaluation of any decreased pigmentation in those 2 animals. After 12 weeks of recovery, there were no AZD3293-related pigmentation effects in males. In females given AZD3293 at $200 \mathrm{mg} / \mathrm{kg} / \mathrm{day}$ only, minimally decreased pigmentation was observed in 1 animal indicating partial recovery at $200 \mathrm{mg} / \mathrm{kg} /$ day and complete recovery at 30 and $100 \mathrm{mg} / \mathrm{kg} /$ day. Also, at this time point, skin sections from a shaved pigmented area were examined in all control and high dose $(200 \mathrm{mg} /$ $\mathrm{kg} /$ day) females and 1 female at $100 \mathrm{mg} / \mathrm{kg} /$ day and no AZD3293-related findings were noted in these shaved skin sections.

Minimal to marked PNL2 positivity (cytoplasmic staining) was observed at all time points in all groups, including control, with a similar incidence and/or grade. PNL2 positive cells were noted in hair follicles, usually lining the dermal papilla and a few scattered positive cells were also present in the basal layer of the epidermis (more numerous in males compared to females) and in the hair sheath. The PNL-2-positive cells were therefore interpreted to be melanocytes. Additionally, the few abnormal pigmented cells observed in H\&E sections were also PNL-2-positive. Based on these results, melanocyte numbers appeared to be similar between control and AZD3293-treated animals at all time points.

\section{Effects of AZD3293 on PMEL processing in human MNT1 cell cultures}

Human MNT1 cells (pigmented melanoma cell line) were used to assess the effects of AZD3293 and other BACE inhibitors on PMEL proteolytic processing. Cells were treated for $24 \mathrm{hr}$ with compounds having different potencies for BACE1 versus BACE2 then analyzed by immunoblotting to detect $M \beta$, cleavage of which is required for melanogenesis. As shown in Figure 5, the BACE1/2 non-selective inhibitor AZD3293 induced a concentration-dependent accumulation of $\mathrm{M} \beta$ beginning at low nanomolar concentrations, consistent with its high potency for inhibiting BACE enzymes. The reference BACE inhibitor compounds AZD3839 and AZ1340 induced an increase in M $\beta$ at concentrations that roughly correlate with their BACE2 $\mathrm{IC}_{50} \mathrm{~s}$ (444 and $>5000$ $\mathrm{nM}$, respectively) but not their BACE1 $\mathrm{IC}_{50} \mathrm{~s}$ (36 and 308 $\mathrm{nM}$, respectively). AZD3293-induced accumulation of $\mathrm{M} \beta$ was also observed in pigmented primary human melanocytes and murine B16F10 cells (data not shown) demonstrating the results in MNT1 cells are not unique to the human melanoma cell line. These results are consistent with other pharmacological, genetic, and siRNA studies indicating a role for BACE2 but not BACE1 in melanocyte PMEL processing $(20,21)$.

\section{Evaluation of the potential de-pigmenting effects of AZD3293 in normal human epidermal melanocytes and melanocyte-containing reconstructed human epithelium}

The potential effects of AZD3293 on melanin synthesis were investigated in both biochemical and cell-based assays. More specifically, effects of AZD3293 were evaluated on the following: activity of human tyrosinase enzyme; melanin synthesis in normal human epidermal melanocytes (NHEM) stimulated by a stable $\alpha-\mathrm{MSH}$ analog (4-norleucine, 7-D-phenylalanine- $\alpha$-melanocytestimulating hormone; NDP-MSH); and melanin synthesis in a pigmented reconstructed human epidermis model (RHEm). The expression of PMEL17 in pigmented reconstructed human epidermis was also evaluated by immunofluorescent labeling. There were no cytotoxic effects caused by AZD3293 tested at up to $30 \mu \mathrm{M}$ nor did it show any inhibitory effect on tyrosinase enzyme activity.

AZD3293, tested to $1 \mu \mathrm{M}$, showed no inhibitory effect on melanin synthesis by NDP-MSH-stimulated melanocytes. In fact, at the highest concentrations, AZD3293 tended to increase melanin synthesis (Figure 6A). Similar to cell culture experiment, treatment of RHEm with AZD3293 up to $10 \mu \mathrm{M}$ for 10 days did not result in significant modulation of the melanin content compared with the control condition. Qualitative and quantitative analysis of PMEL17 labeling showed that treatment of RHEm with AZD3293 up to $1 \mu \mathrm{M}$ for 10 days did not cause any significant changes of PMEL17 expression compared to the control condition. The potential effects of AZD3293 on melanocyte dendricity were evaluated in a co-culture model of NHEM and normal human epidermal keratinocytes in the presence of isobutylmethyl xanthine (IBMX; a melanocyte dendricity stimulating agent). Under the experimental conditions of this assay, AZD3293 did not show any significant effect on IBMX-induced melanocyte dendricity in the melanocyte/ keratinocyte co-culture (Figure 6B).

In conclusion, under the conditions of these experiments AZD3293 had no effects on melanin production, melanocyte viability, or morphology in cultured human melanocytes or reconstituted human epidermis.

\section{Effects in a Phase 1 clinical study}

In Part 2 of the Phase 1 multiple-ascending dose study of AZD3293, skin biopsy samples were obtained 
before and after 13 doses of AZD3293 over 14 days and processed for histological evaluation and assessment of melanin content. Examples of Grade 1 and Grade 4 staining in Stratum basale ( $\mathrm{Sb}$ ) and Stratum spinosum (Ss) are shown in Figure 7, top panel. The general skin morphology of all samples showed normal status, except for one subject in the low dose (15 mg/day) group who in a post-dose sample showed unspecific irritation with slight to moderate sub-epidermal inflammation and epidermal hyper- and parakeratosis. This observation was considered not to be treatment-related. Subsequently, un-blinding of the study data confirmed the consistency in the initial melanin grading as no subject had more than a single step change in melanization grade between epidermal compartments pre- and post-dosing (Figure 7, bottom panel). No gross hypopigmentation of the hair or skin was observed in the appearance of any study subject.

No degenerative effects were observed in epidermis or dermis in connection with 13 doses of AZD3293 given 14 days (up to $150 \mathrm{mg}$ daily). Melanin content was either increased or decreased in the main layers of epidermis in a minority of subjects exposed to AZD3293, but there were no obvious differences when compared to control subject biopsies, nor was there a suggestion of a doseresponse relationship. Content of melanin was generally lower in hair follicles than in epidermis in all study subjects. Only two subjects had hair follicles present in both pre- and post-dose samples. Both were treated with AZD3293 (15 mg and $50 \mathrm{mg}$, respectively) and melanin grade changed from 1 in the pre-dose sample to 0 in the post-dose sample in both subjects. However, 3 subjects in the control group also had hair follicles in either pre- or post-dose biopsies and the melanization grade in their hair follicles was 0,0 , and 1 , respectively, suggesting that the melanization grade in all subject hair follicles was very low to begin with. Four subjects receiving AZD3293 (two from the $15 \mathrm{mg}$ group and one each from $50 \mathrm{mg}$ and $150 \mathrm{mg}$ groups) had hair follicles present in their post-dose biopsies. The melanization grade in their hair follicles was 1, 0, 1, and 0, respectively. In aggregate, there were no significant changes in hair follicle melanin content between control and AZD3293-treated subjects.

In conclusion, due to variation in melanization in the control and AZD3293-treatment group biopsies, the minimal nature of changes, and the lack of dosedependent trends, we conclude that AZD3293 had no significant effect on the melanin content in hair follicles or epidermal melanization in subjects who received 13 doses of AZD3293 over 14 days at dose levels of up to $150 \mathrm{mg}$. Clearly data from skin examinations in the longer-term ongoing Phase 3 studies will be more definitive with respect to the translatability of preclinical hypopigmentation findings.

\section{Discussion}

\section{Depigmentation observed in dogs and Long- Evans rats was dose-dependent and reversible}

No pigmentation changes or any other skin changes were noted in any of the short-term toxicology studies in the dog, including two 14-day dose range-finding studies and the 28-day toxicology study (data not reported here). In the 13-week and 39-week dog toxicology studies however, AZD3293 produced pronounced hypopigmentation of fur-less skin, oral mucosa, and dark fur (but not tan-colored fur) in all animals, with no apparent dose relationship to severity, which suggests that in these two studies the lowest doses were sufficient for a full depigmentation effect. This is not entirely surprising given that the maximum unbound plasma AZD3293 exposure at the low dose in the 3-month dog toxicology study ( $1 \mathrm{mg} / \mathrm{kg} /$ day) was $230 \mathrm{nM}$, which is more than 200-fold over the BACE2 IC I0 $_{50}$ for AZ3293. Even assuming that free plasma AZD3293 exposure may not represent the actual concentration of AZD3293 at the target, it is safe to assume that the $1 \mathrm{mg} / \mathrm{kg}$ dose gave a full suppression of the melanocyte-expressed BACE2 activity. In the 13-week study in older dogs no true no-effect level could be established either, but unlike the two previous studies at higher doses, a clear dosedependency of the depigmentation was seen and a dose level with only minimal changes could be established. Thus, even though minimal changes were seen at $0.1 \mathrm{mg} /$ $\mathrm{kg} /$ day, there was an increased severity and more rapid onset of decreased pigmentation observed at doses over $1 \mathrm{mg} / \mathrm{kg} /$ day. Similarly, in animals treated weekly with AZD3293, there was an increased severity of decreased pigmentation at $5 \mathrm{mg} / \mathrm{kg} /$ week compared to the lower doses. Moreover, weekly dosing per se resulted in a lower severity of findings as compared to daily dosing, which further emphasizes that the depigmentation effects of AZD3293 are dose-dependent.

In pigmented Long-Evans rats, daily dosing with AZD3293 produced clearly dose-dependent depigmentation. Thus, in animals treated with the lowest dose $\left(30 \mathrm{mg} / \mathrm{kg} /\right.$ day; resulting in a mean $\mathrm{C}_{\max }$ that was 158-fold higher than the BACE2 $\mathrm{IC}_{50}$ ) the depigmentation was considerably less prominent at all stages of the study compared to groups treated with 100 or $200 \mathrm{mg} / \mathrm{kg} /$ day.

In contrast to data reported by Shimshek, et al. (20) using various mouse models, our data from pigmented rat and dog studies show that the depigmentation induced by AZD3293 is reversible. In the dog studies, the histopathological changes in the hair follicle and epidermis had fully recovered by the end of the 8-week or 13-week dose-free periods following the 3-month and 9-month dosing, respectively. There was also partial macroscopic recovery of the pigmentation of the skin and fur by the end of the corresponding recovery periods. 
The striped-pattern appearance of some of the facial hair straws in dogs dosed weekly also suggests rapid functional recovery of normal melanin production and melanosome export onto keratinocytes as soon as the pharmacological inhibition of BACE2 had ceased. This was seen throughout the 9-month study suggesting that eumelanin-producing machinery is largely intact despite chronic BACE2 inhibition.

In Long-Evans rats, partial recovery of skin pigmentation was observed after 4 weeks following cessation of AZD3293 dosing and complete recovery was seen at $30 \mathrm{mg} / \mathrm{kg} /$ day after 12 weeks. Microscopically, partial recovery of skin findings at $200 \mathrm{mg} / \mathrm{kg} /$ day was seen as early as 1 week into recovery and complete microscopic recovery was observed at $200 \mathrm{mg} / \mathrm{kg} /$ day in males after 4 weeks of recovery and at $\leq 100 \mathrm{mg} /$ $\mathrm{kg} /$ day in females after 12 weeks of recovery, which correlated with the partial recovery of the pale fur observed macroscopically. These data strongly suggest that a complete recovery of black pigmentation would have been achieved even at the higher doses had the recovery period been extended beyond 12 weeks.

\section{BACE2 inhibition of PMEL processing is required for AZD3293-induced depigmentation}

Biochemical studies in human skin preparations established that AZD3293 did not affect melanin synthesis per se; it had no effect on regulatory mechanisms of melanin synthesis and it was not cytotoxic to cultured human melanocytes. Our data suggests that BACE2 and not BACE1 inhibition is the necessary condition to interrupt PMEL processing because a BACE1-selective inhibitor, at least within the limits of its BACE2 selectivity, did not have any effect on PMEL processing in vitro. Furthermore, the same BACE1selective compound did not induce any changes in skin or fur pigmentation when given daily for three months to Beagle dogs at a mean $C_{\max }$ that was 14-fold higher than the BACE1 $\mathrm{IC}_{50}$ and approximately equal to the BACE2 $\mathrm{IC}_{50}$. AZD3293 is almost equipotent at both BACE1 and BACE2 enzymes and so it appears that it is necessary to inhibit BACE2 in melanocytes to preclude processing of melanocyte-specific protein, PMEL, thus effectively removing the scaffold around which eumelanin is normally packaged within the melanosome, and ultimately resulting in less eumelanin being transferred to keratinocytes and hair shaft leading to depigmentation in animals $(21,33)$.

As described above, hair follicle melanocytes changed their appearance (they were filled with more numerous, rounder and larger melanosomes) following AZD3293 treatment. Similar intracellular structures with irregular pigment deposits have been observed following ApoE, which is important in PMEL sorting onto melanosomes, depletion in melanocytes [40] suggesting that any process related to PMEL processing and PMEL-fibril formation might result in similar melanosome morphology changes.

\section{Clinical experience with AZD3293 to date and potential for human translation of the depigmentation}

Hypopigmentation by systemic administration of pharmaceutical agents is an extremely rare finding in nonclinical safety studies. Whether the degree to which depigmentation such as was observed in dog and rat would be expected to translate into humans is difficult to assess because of the scarcity of data. Therefore, the longterm significance of the animal depigmentation findings for human safety assessment is currently unknown. It is worth noting, however, that certain aspects of human and animal skin and hair pigmentation (such as synchronicity and duration of hair cycle, location of melanin granules within the hair shaft, etc.) are quite different, in particular with respect to hair pigmentation of human scalp hair and that of short-haired animals (such as rodents and Beagle dogs) (41). For instance, in humans the melanin production is continuous throughout the lifetime of any individual hair shaft (up to 5 years), whereas in rats and dogs active melanin production in hair follicles is measured in weeks followed by a long dormant phase (42).

No depigmentation effects were noted in humans in the Phase 1 multiple-dose clinical study in Alzheimer's disease patients treated with AZD3293 over 14 days at doses that achieved exposures which exceeded those observed at the lowest effect dose levels for depigmentation in the animal studies. Moreover, there were no changes indicative of eumelanin reduction at a cellular level in skin biopsies in human subjects treated daily with doses roughly equivalent to doses that produced very clear changes in dog hair follicle melanocytes after just one week of dosing. It should be noted, however, that the number of subjects in the human multiple ascending dose study may be too low, the dosing duration may have been too short, and the dynamic range for the melanin scoring may have been too small to draw unequivocal conclusions.

In aggregate, these data suggest that BACE inhibitorproduced depigmentation in animal studies may not translate in humans, or at the very least, that humans are less sensitive to this effect. It is unlikely that these sensitivity differences are due to differences in affinity of AZD3293 to rat, dog, and human BACE2 because the 24 amino acid residues that define the BACE2 ligand binding site (18) are identical across these species. Formally testing this hypothesis would require recombinant BACE2 assays for each species, which currently are not available. Obviously, the ongoing clinical studies with AZD3293 and other non-selective BACE1 / BACE2 inhibitors in clinical development will either prove or disprove any assumptions regarding the 
clinical translatability of depigmentation made on the basis of animal and/or in vitro studies alone.

\section{Overall conclusions}

In conclusion, AZD3293-induced depigmentation was animal age- and dose-dependent occurring from the $5^{\text {th }}-6^{\text {th }}$ week of treatment, and was readily reversible following 4- or 13-week treatment-free recovery periods. The reversibility of these findings differs from previously reported in vivo depigmentation effects of BACE inhibition in several mice strains (20) where no spontaneous recovery was seen up to 100 days following cessation of BACE inhibitor treatment. The reasons for this discrepancy are not clear but could be related to the long melanocyte retention time of the BACE inhibitor used by Shimshek, et al. (20) because, according to the authors, hair color did return to normal once the depigmented hair had been plucked, which presumably induced a new proliferation phase in the hair follicles. Thus, the apparent lack of recovery observed by Shimshek, et al. (20) could be compound- rather than class-specific.

Depigmentation induced by AZD3293 did not affect pigmented tissues other than hair-less skin, hair, and oral mucosa (which was only assessed in the dog) and was not accompanied by melanocyte death or any degenerative or inflammatory changes in these tissues in either species. Specifically, there were no hypopigmentation effects in Substantia Nigra, inner ear, or retinal pigmented epithelium. The appearance of the animals correlated with marked decrease of eumelanin levels in the epidermal and hair follicle keratinocytes and changes in the morphological appearance of melanocytes. Of note, although some abnormal, hypertrophic, and pigmented melanocytes were seen in some of the hair follicles from both rats and dogs dosed daily with AZD3293 the melanocyte numbers in hair follicles appeared to be similar to control levels. Importantly, only eumelanin was affected while production of pheomelanin (the redbrown pigment) was unchanged, as shown by the lack of depigmentation of the tan-colored fur in Beagle dogs even after 9 months of daily dosing. At similar exposures, depigmentation appeared more extensive in dogs than in rats, as effects dogs occurred at about 5-fold lower plasma AZD3293 exposures compared to rats.

Depigmentation was clearly correlated with BACE2 inhibition, and BACE1 inhibition alone was insufficient to produce depigmentation either in vitro or in vivo. Thus, in agreement with previous findings, BACE2 inhibition appeared to produce depigmentation by interfering with the processing of PMEL, a fragment of which is the primary component of protein scaffolding necessary for correct packaging of newly synthesized eumelanin in melanosomes. Such scaffold-less melanosomes apparently do not mature normally even if eumelanin synthesis per se is not blocked. However, as shown by
EM data, melanosome size, shape, and contents appear to be affected, which seems to preclude their transfer from melanocytes to keratinocytes in the epidermis or into hair shaft in the hair follicle. It is the deficit in fresh melanosome "supply" to the newly differentiated keratinocytes and the growing hair shaft that eventually produces gradual depletion of eumelanin levels in these skin structures.

AZD3293 did not have any effect on melanin synthesis, cell viability, or morphology in human primary melanocytes and reconstituted human epidermis exposed to concentrations of AZD3293 exceeding the BACE2 $\mathrm{IC}_{50}$ by 1000 -fold or more. Furthermore, AZD3293 did not cause any significant changes in melanization or the appearance of melanocytes in skin biopsies taken from human subjects treated daily with supra-therapeutic doses of AZD3293 over 14 days (the highest dose being studied in ongoing Phase 3 studies is $50 \mathrm{mg} /$ day). Plasma AZD3293 exposures in human subjects were similar to the exposures in the animal studies at which depigmentation at the cellular level was clearly present after just one week of treatment (and some of the hair pigmentation changes in the dog, such as the striped-pattern appearance of some of the facial hair, were observed even after a single dose). These data suggest that the depigmentation findings observed in animals may have poor translatability to humans. Based on the results of our studies we hypothesize that various animal species and humans are not equally sensitive to BACE2 inhibition with respect to effects on eumelanin production, with dogs being the most sensitive and humans the least sensitive species. Reasons for this apparent species-specific sensitivity are not clear but could be related to subject age and species differences in hair cycles, hair follicle morphology, and melanocyte function.

\section{Funding: All studies described in this paper were funded by AstraZeneca.}

Acknowledgements: The authors gratefully acknowledge the contributions of many colleagues at AstraZeneca and Eli Lilly \& Co., Inc. who worked on these studies and/or the interpretation/description of the findings, especially John Sims, MD; Chris Aluise, PhD; Scott Monk, PhD; Brian Willis, PhD, Craig Shering, DPhil; and Jamie Mullen, MD for their reviews and constructive comments on early drafts of this manuscript.

Author disclosures: - Gvido Cebers, MD, PhD: GC was an AstraZeneca employee at the time the primary manuscript work was completed. GC is no longer an employee of AstraZeneca. GC has no financial disclosures. - Typhaine Lejeune, DVM, DECVP: TL is an employee of Charles River Laboratories, which conducted in vivo non-clinical studies reported in this paper as a paid contractor for AstraZeneca. TL has no financial disclosures. - Bassem Attalla, BS: Dr. BA is an employee of Charles River Laboratories, which conducted in vivo non-clinical studies reported in this paper as a paid contractor for AstraZeneca. BA has no financial disclosures. - Magnus Soderberg, MD, PhD: MS is an employee of AstraZeneca and owns AstraZeneca stock. MS has no other financial disclosures. - Robert C. Alexander, MD: RCA was an AstraZeneca employee at the time the primary manuscript work was completed. RCA is no longer an employee of AstraZeneca but remains a participant in AstraZeneca's long term incentive program and owns AstraZeneca stock. RCA is currently an employee of Pfizer. RCA has no other financial disclosures. - Samantha Budd Haeberlein, PhD: $\mathrm{SBH}$ was an AstraZeneca employee at the time the primary manuscript work was completed. SBA is no longer an employee of AstraZeneca but remains a participant in AstraZeneca's long term incentive program and owns AstraZeneca stock. SBA is currently an employee of Biogen. SBA has no other financial disclosures. - Alan R. Kugler, PhD: ARK was an AstraZeneca employee at the 
time the primary manuscript work was completed. ARK is no longer an employee of AstraZeneca but remains a participant in AstraZeneca's long term incentive program and owns AstraZeneca stock. ARK has no other financial disclosures. • Evan W. Ingersoll, PhD: EWI was a paid independent contractor for AstraZeneca during the time these studies were conducted and throughout this manuscript development. He provided program management, scientific support, and medical writing services including serving as coordinating editor for this manuscript. EWI has no other financial disclosures. - Stefan Platz, DVM, PhD: SP is an AstraZeneca employee, is a participant in AstraZeneca's long term incentive program, and owns AstraZeneca stock. SP has no other financial disclosures. - Clay W. Scott, $\mathrm{PhD}$ : CWS is an AstraZeneca employee, is a participant in AstraZeneca's long term incentive program, and owns AstraZeneca stock. CWS has no other financial disclosures.

Ethical standards: All procedures involving the care and use of animals in these studies were reviewed and approved by the Test Facility's Institutional Animal Care and Use Committee before conduct. During these studies, the care and use of animals were conducted in accordance with the guidelines of the USA National Research Council and the Canadian Council on Animal Care. The protocol for the Phase 1 study described here was approved by the independent Institutional Review Board, Aspire IRB, Santee, CA, USA. The studies were performed in accordance with the Declaration of Helsinki of 1975 and International Conference on Harmonisation/Good Clinical Practice guidelines. All subjects provided written informed consent prior to enrollment into the studies.

\section{References}

1. Younkin SG. Evidence that A beta 42 is the real culprit in Alzheimer's disease. Ann Neurol 1995;37, 287-288.

2. Steiner H, Capell A, Leimer U, Haass C. Genes and mechanisms involved in beta-amyloid generation and Alzheimer's disease. Eur Arch Psychiatry Clin Neurosci 1999;249, 266-270.

3. Iwatsubo T, Odaka A, Suzuki N, Mizusawa H, Nukina N, Ihara Y. Visualization of A beta $42(43)$ and A beta 40 in senile plaques with endspecific A beta monoclonals: evidence that an initially deposited species is A beta 42(43). Neuron 1994;13, 45-53.

4. Vassar R, Bennett BD, Babu-Khan S, Kahn S, Mendiaz EA, Denis P, et al.. Beta-secretase cleavage of Alzheimer's amyloid precursor protein by the transmembrane aspartic protease BACE. Science 1999;286, 735-741.

5. Karran E, Mercken M, De Strooper B. The amyloid cascade hypothesis for Alzheimer's disease: an appraisal for the development of therapeutics. Nat Rev Drug Discov 2011;10, 698-712.

6. Citron M, Oltersdorf T, Haass C, McConlogue L, Hung AY, Seubert P, et al. Mutation of the beta-amyloid precursor protein in familial Alzheimer's disease increases beta-protein production. Nature 1992;360, 672-674.

7. Serneels L, Van Biervliet J, Craessaerts K, Dejaegere T, Horre K, Van Houtvin $\mathrm{T}$, et al. gamma-Secretase heterogeneity in the Aph1 subunit: relevance for Alzheimer's disease. Science 2009;324, 639-642.

8. Jonsson T, Atwal JK, Steinberg S, Snaedal J, Jonsson PV, Bjornsson S, et al A mutation in APP protects against Alzheimer's disease and age-related cognitive decline. Nature 2012;488, 96-99.

9. McConlogue L, Buttini M, Anderson JP, Brigham EF, Chen KS, Freedman SB, et al. Partial reduction of BACE1 has dramatic effects on Alzheimer plaque and synaptic pathology in APP Transgenic Mice. J Biol Chem 2007;282, 2632626334.

10. Sankaranarayanan S, Holahan MA, Colussi D, Crouthamel MC, Devanarayan $\mathrm{V}$, Ellis J, et al. First demonstration of cerebrospinal fluid and plasma A beta lowering with oral administration of a beta-site amyloid precursor proteincleaving enzyme 1 inhibitor in nonhuman primates. J Pharmacol Exp Ther 2009;328, 131-140.

11. May PC, Dean RA, Lowe SL, Martenyi F, Sheehan SM, Boggs LN, et al. Robust central reduction of amyloid-beta in humans with an orally available, non-peptidic beta-secretase inhibitor. J Neurosci 2011;31, 16507-16516.

12. May PC, Willis BA, Lowe SL, Dean RA, Monk SA, Cocke PJ, et al. The potent BACE1 inhibitor LY2886721 elicits robust central Abeta pharmacodynamic responses in mice, dogs, and humans. J Neurosci 2015;35, 1199-1210.

13. Menting KW, Claassen JA. beta-secretase inhibitor; a promising novel therapeutic drug in Alzheimer's disease. Front Aging Neurosci 2014;6, 165.

14. Vassar R. BACE1 inhibitor drugs in clinical trials for Alzheimer's disease. Alzheimers Res Ther 2014;6, 89.

15. Yan R, Vassar R. Targeting the beta secretase BACE1 for Alzheimer's disease therapy. Lancet Neurol 2014;13, 319-329.

16. Eketjall S, Janson J, Kaspersson K, Bogstedt A, Jeppsson F, Falting J, et al AZD3293: A Novel, Orally Active BACE1 Inhibitor with High Potency and Permeability and Markedly Slow Off-Rate Kinetics. J Alzheimers Dis 2016;50, 1109-1123.

17. Stutzer I, Selevsek N, Esterhazy D, Schmidt A, Aebersold R, Stoffel M. Systematic proteomic analysis identifies beta-site amyloid precursor protein cleaving enzyme 2 and 1 (BACE2 and BACE1) substrates in pancreatic betacells. J Biol Chem 2013;288, 10536-10547.

18. Mirsafian H, Mat Ripen A, Merican AF, Bin Mohamad S. Amino acid sequence and structural comparison of BACE1 and BACE2 using evolutionary trace method. ScientificWorldJournal 2014, 482463.

19. Sun X, He G, Song W. BACE2, as a novel APP theta-secretase, is not responsible for the pathogenesis of Alzheimer's disease in Down syndrome. FASEB J 2006;20, 1369-1376.

20. Shimshek DR, Jacobson LH, Kolly C, Zamurovic N, Balavenkatraman KK, Morawiec L, et al. Pharmacological BACE1 and BACE2 inhibition induces hair depigmentation by inhibiting PMEL17 processing in mice. Sci Rep 2016;6, 21917.

21. Rochin L, Hurbain I, Serneels L, Fort C, Watt B, Leblanc P, et al. BACE2 processes PMEL to form the melanosome amyloid matrix in pigment cells. Proc Natl Acad Sci U S A 2013;110, 10658-10663.

22. Kerje S, Sharma P, Gunnarsson U, Kim H, Bagchi S, Fredriksson R, et al. The Dominant white, Dun and Smoky color variants in chicken are associated with insertion/deletion polymorphisms in the PMEL17 gene. Genetics 2004;168, 1507-1518.

23. Clark LA, Wahl JM, Rees CA, Murphy KE. Retrotransposon insertion in SILV is responsible for merle patterning of the domestic dog. Proc Natl Acad Sci U S A 2006;103, 1376-1381.

24. Brunberg E, Andersson L, Cothran G, Sandberg K, Mikko S, Lindgren G. A missense mutation in PMEL17 is associated with the Silver coat color in the horse. BMC Genet 2006;7, 46.

25. Sturm RA, Teasdale RD, Box NF. Human pigmentation genes: identification structure and consequences of polymorphic variation. Gene 2001;277, 49-62.

26. Kobayashi T, Urabe K, Orlow SJ, Higashi K, Imokawa G, Kwon BS, et al. The Pmel 17/silver locus protein. Characterization and investigation of its melanogenic function. J Biol Chem 1994;269, 29198-29205.

27. Lee ZH, Hou L, Moellmann G, Kuklinska E, Antol K, Fraser M, et al. Characterization and subcellular localization of human Pmel 17/silver, a $110-\mathrm{kDa}$ (pre)melanosomal membrane protein associated with 5,6,-dihydroxyindole-2-carboxylic acid (DHICA) converting activity. J Invest Dermatol 1996;106, 605-610.

28. Ito S, Wakamatsu K. Chemistry of mixed melanogenesis--pivotal roles of dopaquinone. Photochem Photobiol 2008;84, 582-592.

29. Furumura M, Sakai C, Potterf SB, Vieira WD, Barsh GS, Hearing VJ. Characterization of genes modulated during pheomelanogenesis using differential display. Proc Natl Acad Sci U S A 1998;95, 7374-7378.

30. Berson JF, Harper DC, Tenza D, Raposo G, Marks MS. Pmel17 initiates premelanosome morphogenesis within multivesicular bodies. Mol Biol Cell 2001;12, 3451-3464.

31. Watt B, van Niel G, Raposo G, Marks MS. PMEL: a pigment cell-specific model for functional amyloid formation. Pigment Cell Melanoma Res 2013;26, 300-315.

32. Theos AC, Berson JF, Theos SC, Herman KE, Harper DC, Tenza D, et al. Dual loss of ER export and endocytic signals with altered melanosome morphology in the silver mutation of Pmel17. Mol Biol Cell 2006;17, 35983612 .

33. van Bebber F, Hruscha A, Willem M, Schmid B, Haass C. Loss of Bace2 in zebrafish affects melanocyte migration and is distinct from Bace1 knock out phenotypes. J Neurochem 2013;127, 471-481.

34. Jeppsson F, Eketjall S, Janson J, Karlstrom S, Gustavsson S, Olsson LL, et al. Discovery of AZD3839, a potent and selective BACE1 inhibitor clinical candidate for the treatment of Alzheimer disease. J Biol Chem 2012;287, 41245-41257.

35. Poumay Y, Coquette A. Modelling the human epidermis in vitro: tools for basic and applied research. Arch Dermatol Res 2007;298, 361-369.

36. Poumay Y, Dupont F, Marcoux S, Leclercq-Smekens M, Herin M, Coquette A. A simple reconstructed human epidermis: preparation of the culture model and utilization in in vitro studies. Arch Dermatol Res 2004;296, 203-211.

37. Alexander R, Rosen L, Russell M, Kugler AR, Cebers G, Ye N, et al. AZD3293 A Novel BACE1 Inhibitor: Pharmacokinetics and Effects on Plasma and CSF A $\beta$ Peptides Following Multiple-Dose Administration in Alzheimer's Disease Patients. Neurodegener Dis, 2015;702.

38. Cebers G, Alexander RA, Budd Haeberlein S, Han D, Goldwater R, Ereshefsky L, et al. (Accepted for Publication) AZD3293: Pharmacokinetic and pharmacodynamic effects in healthy subjects and patients with Alzheimer's disease. J Alzheimers Dis.

39. Hellstrom AR, Watt B, Fard SS, Tenza D, Mannstrom P, Narfstrom K, et al Inactivation of Pmel alters melanosome shape but has only a subtle effect on visible pigmentation. PLoS Genet 2011;7, e1002285.

40. van Niel G, Bergam P, Di Cicco A, Hurbain I, Lo Cicero A, Dingli F, et al. Apolipoprotein E Regulates Amyloid Formation within Endosomes of Pigment Cells. Cell Rep 2015;13, 43-51.

41. Tobin D. The cell biology of human hair follicle pigmentation. Pigment Cell Melanoma Res 2011;24, 75-88.

42. Thibaut S, de Becker E, Bernard BA, Huart M, Fiat F, Baghdadli N, et al. Chronological ageing of human hair keratin fibres. Int J Cosmet Sci 2010;32, 422-434. 